IMA Journal of Applied Mathematics (2018) Page 1 of 26 doi:10.1093/imamat/xxx000

\title{
An elastohydrodynamical simulation study of filament and spermatozoan swimming driven by internal couples
}

\author{
Kenta IsHIMOTO* \\ Wolfson Centre for Mathematical Biology, Mathematical Institute, University of Oxford, \\ Oxford OX2 6GG, UK \\ Graduate School of Mathematical Sciences, The University of Tokyo, Tokyo 153-8914, Japan \\ *Corresponding author: ishimoto@maths.ox.ac.uk \\ AND \\ EAMONN A. GAFFNEY \\ Wolfson Centre for Mathematical Biology, Mathematical Institute, University of Oxford, \\ Oxford OX2 6GG, UK
}

[Received on 26 April 2018]

\begin{abstract}
Eukaryotic cell swimming is frequently actuated via the flagellum, which is a slender flexible appendage driven by waves of internal couples generated by dynein molecular motors. Here we adapt a regularised elastohydrodynamic model of flagella by Simons et al. (2015) to consider active filament models of sperm swimming given the patterns of dynein couples indicated by the analysis of flagellate digital microscopy, with a further consideration of helically beating flagella. We additionally consider whether boundary accumulation and rheotaxis, as predicted by modelling studies with prescribed flagellum waveforms, are inherited once flagellum elasticity is accommodated with the flagellum deforming in response to the mechanical forces it experiences. However the simulations presented here are limited to filament morphologies and thus either headless sperm or those with a filamentous head. We find that simple patterns of dynein contraction generate flagellar waveforms that are qualitatively similar to observation and also that flagellum buckling instabilities predicted by resistive force theory elastohydrodynamical models need not occur. Furthermore, modelling simulations with prescribed helical waveforms qualitatively match elastohydrodynamic modelling predictions in the context of boundary accumulation but flagellum elasticity is predicted to ameliorate the impact of background flows for flagellates with a helically beating flagellum near a surface. In contrast, elastohydrodynamic modelling predictions for boundary accumulating flagellates with planar waveforms indicate that boundary behaviours are subtle for these cells and fully reproducing observed behaviours, while accommodating dynamic flagellar deformation, raises further modelling challenges in the study of swimming cells.
\end{abstract}

Keywords: Elastohydrodynamics. Flagellate Swimming. Boundary Accumulation. Rheotaxis.

\section{Introduction}

Virtually all sperm, and many diverse protists, swim via the movement of the eukaryotic flagellum, an exquisite nanobioengineered motile filament, which is conserved across numerous diverse cells. Its structure has been extensively reported (Fawcett, 1975), with nine outer microtubule doublets concentric to a central pair of microtubules, with extensive connecting structures, in particular radial spokes which mechanically reinforce the filament in its radial direction and nexin links, which reinforce it tangentially. This structure, of nine microtubule doublets and a central pair, is also known as the 9+2 axoneme. 

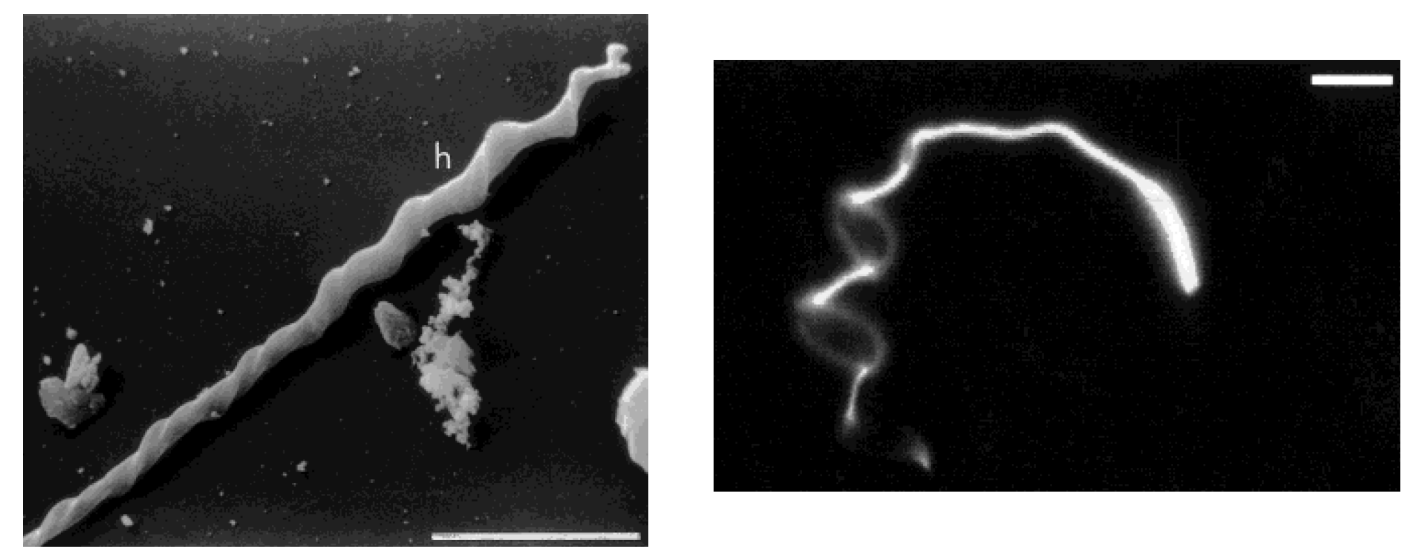

FIG. 1. Left. An electronmicrograph of a starling sperm head (h) and proximal flagellum emphasising the thin, extended geometry of the sperm head. Scale bar: 1 micron. Right. A pigeon sperm, with a 3D helical beat pattern and an elongated sperm head, tightly circling adjacent to an overlying coverslip. Scale bar: 10 microns. Figures reproduced with permission from Vernon \& Woolley (1999).

Often this is further reinforced in a species-specific manner as exemplified by mammalian sperm, which exhibit outer dense fibres running alongside the microtubule doublets and an anisotropic fibrous sheath, all of which taper from proximal to distal, that is towards the flagellum tip (Fawcett, 1975).

All such structures are passive. In addition molecular motors, referred to as dyneins, act to slide the microtubule doublets relative to each other (Brokaw, 1971, 1972; Fawcett, 1975). However, with the flagellum abutting the cell at the proximal end of the flagellum, where relative sliding of the microtubules is inhibited, the dyneins do not induce microtubule sliding, but instead microtubule bending. One particularly elegant illustration of this arises in Lindemann et al.'s (1992) experiments, where dynein motor action was induced for partially digested and decapitated rat sperm, with the result that the flagella separated by the action of groups of microtubule doublets sliding relative to each other.

Amplitude modulated travelling waves of internal torque, first in one direction and then the opposite, have been indicated in planar beating human sperm (Gaffney et al., 2011). These in turn bend the flagellum back and forth. When coupled with the viscous resistance of the surrounding fluid, which reflects the anisotropy of a filament, whereby the flagellum experiences greater drag for movement perpendicular to its central axis than along its central axis, a net movement of the flagellum, and thus the cell, is generated. Detailed mechanical explanations, with experimental validation, were developed by Gray \& Hancock (1955), while a more intuitive but qualitative explanation may be found in Ishimoto \& Gaffney (2015b).

In addition, more complex three dimensional movements of the flagellum are also commonly observed (Ishijima et al., 1992; Vernon \& Woolley, 1999; Woolley \& Vernon, 2001; Woolley, 2003), highlighting that the regulation of the dynein contractions can also be spatially complex. However, such regulation is difficult to experimentally assess, and hence modelling has been instrumental in developing and testing several competing hypotheses for dynein behaviour, for instance curvature control (Brokaw, 1972, 2002, 2009), excitable dynamics, (Murase et al., 1989) sliding control (Camalet \& Julicher, 2000; Riedel-Kruse et al., 2007) and geometrical clutching (Lindemann, 1994b,a, 2002; Bayly \& Wilson, 
2014, 2015). However, the fact that there are so many competing hypotheses also highlights that a physical understanding of the flagellum is far from complete.

On analysing such models in detail, it is also clear that the theory of active elastic filaments in viscous fluid is at best considered in the context of resistive force theory (Gray \& Hancock, 1955), an approximation for the drag on a filament in a viscous fluid. Additional modelling studies, examining other aspects of flagellum elasticity and viscous fluid dynamics, have also used this approximation (for example Fu et al. (2008); Gadêlha et al. (2010); Spagnolie \& Lauga (2010); Montenegro-Johnson et al. (2015); Coy \& Gadêlha (2017)). However, while numerous studies have demonstrated that resistive force theory can be accurate for sperm, as illustrated by Johnson \& Brokaw's study (1979), resistive force theory is not suitable for near-surface swimming (Ishimoto \& Gaffney, 2014), multiple flagella or large cell bodies (Johnson \& Brokaw, 1979), as observed with flagellated protists.

Hence, there is extensive interest in coupling elastic filament mechanics and viscous fluid dynamics for flagella more accurately than resistive force theory allows. An influential model for passive filaments was developed by Tornberg \& Shelley (2004), while early active 2D sheet representations of flagella via immersed boundary methods were considered by Fauci \& McDonald (1995) and Dillon et al. (2006), with Elgeti et al. (2010) using a particle based model with elastic flagellum responses based on a rod and spring representation of the flagellum. More recently the integration of filament dynamics (Lim et al., 2008) and regularised Stokeslets (Cortez, 2001) has led to an easily implementable algorithm for the dynamics of an active filament in a viscous fluid (Olson et al., 2013), which has subsequently been used to explore sperm elastohydrodynamics (Simons et al., 2015), assuming a preferred curvature of the flagellum, a form of curvature control. While the use of regularised Stokeslets entails compromising numerical accuracy at the level of the regularisation coefficient analogous to, but not as well-documented as, finite grid size errors in many computational algorithms, the accuracy is still greatly improved compared to the use of resistive force theory. However, the elastohydrodynamic swimmer algorithm of Simons et al. (2015) is, in practice, restricted to the consideration of filaments, and thus our attention below will focus on filament morphologies. This can include active filaments, headless sperm and various piscine and avian sperm with filamentous heads, such as sturgeon (Psenicka et al., 2007) and, as illustrated in Fig. 1, starling and pigeon (Vernon \& Woolley, 1999).

In this context, our objective in this study is to firstly consider how active filament representations of such swimmers behave given travelling waves of internal torque generation, as motivated by the observations of Gaffney et al. (2011). We will also consider helical waves of internal torque generation and how this influences swimming behaviour and we will proceed to consider how such virtual sperm are predicted to behave near a no-slip surface, to assess whether flagellum elasticity is important in the subtle dynamics of sperm boundary accumulation.

In particular, the tendency of sperm to stay near a surface has been subject to extensive observation (Rothschild, 1963; Woolley, 2003) and numerous modelling studies to understand its mechanical basis, though with a prescribed flagellar beat pattern relative to the cell head (Smith et al., 2009a; Ishimoto \& Gaffney, 2014, 2016). Analogous comments also apply for rheotaxis, which is the tendency of sperm to reorientate parallel to external flows (Winet et al., 1984; Miki \& Clapham, 2013; Ishimoto \& Gaffney, 2015a). Consequently, the study of boundary accumulation and rheotaxis below is motivated by the fact that the impact of flagellar elasticity on the emergent beat pattern has not been commonly investigated for these behaviours. One exception though is a study considering resistive force theory models of sperm behaviour near a back-step (Montenegro-Johnson et al., 2015) and a second concerns a particle-based method (Elgeti et al., 2010), where swimming sperm are subject to extensive fluctuations (Smith et al., 2011). 


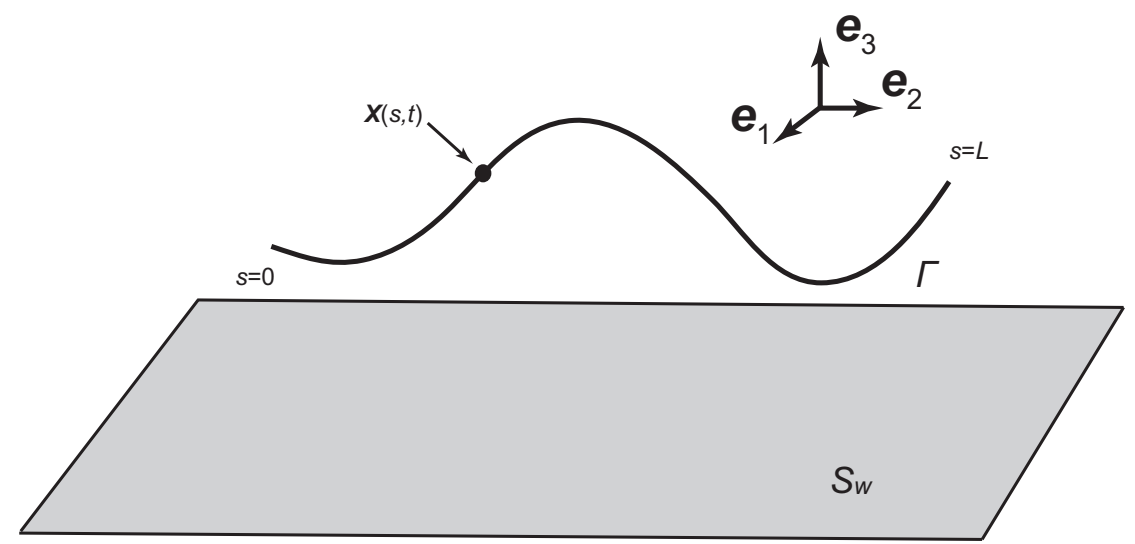

FIG. 2. A schematic picture of an elastic flagellum swimming near an infinite rigid wall, $S_{w}$. The flagellum is represented by a curve $\boldsymbol{X} \in \Gamma$ immersed in a three-dimensional space, whose inertial reference frame basis vectors are denoted by $\left\{\boldsymbol{e}_{1}, \boldsymbol{e}_{2}, \boldsymbol{e}_{3}\right\}$. The curve is parameterised by the initial arclength $s \in[0, L]$ at the initial time.

\section{Modelling Framework}

\subsection{Elastic flagellum}

We consider an elastic filament representation for the sperm flagellum with length $L$ at initial time, and which allows bending, twist and extension but not shear, as proposed by Lim et al. (2008). The filament, $\Gamma$, is denoted by a space curve $\boldsymbol{X}(s, t)$, where $t$ denotes time and $s \in[0, L]$ is a Lagrangian parameter, together with an associated orthonormal triad of directors $\left\{\boldsymbol{D}^{1}(s, t), \boldsymbol{D}^{2}(s, t), \boldsymbol{D}^{3}(s, t)\right\}$, where as commonplace, $\boldsymbol{D}^{3}(s, 0)$ is aligned tangentially along the filament at initial time. Analogously, we take $s$ to be the arclength along the filament at initial time but, as filament extension is allowed, this need not be the arclength for general time.

The filament contact force and contact moment are denoted by $\boldsymbol{F}(s, t)$ and $\boldsymbol{M}(s, t)$, so that the force and torque balances along the filament are given by

$$
\begin{aligned}
\boldsymbol{f}^{e x t}+\boldsymbol{f}^{h y d}+\frac{\partial \boldsymbol{F}}{\partial s} & =\mathbf{0} \\
\boldsymbol{m}^{i n t}+\boldsymbol{m}^{h y d}+\frac{\partial \boldsymbol{M}}{\partial s}+\frac{\partial \boldsymbol{X}}{\partial s} \times \boldsymbol{F} & =\mathbf{0} .
\end{aligned}
$$

Here the term $\boldsymbol{m}^{\text {int }}$ denotes the internal couple per unit of initial length exerted on the filament by the internal dyneins and appeal to Newton's third law reveals that there is no associated force term. The contribution $f^{e x t}$ is the external force per unit of initial length due to external bodies and boundaries, except the surrounding fluid; here it is exclusively due to a wall potential when a no-slip boundary at $z=0$ is considered, as denoted by $S_{w}$ in Fig. 2. There is no associated external moment term, which would have inherited the nomenclature $\boldsymbol{m}^{\text {ext }}$, since the force balance, Eqn. (2.1), has already been used to simplify the full moment balance to arrive at Eqn. (2.2). The hydrodynamic term $f^{\text {hyd }}$ is the force per unit of initial length exerted by the fluid on the filament which, by Newton's third, is minus the force exerted by the filament on the fluid, and hence there is an overall minus sign in Eqns. (2.13)-(2.16) below. In particular note that $\boldsymbol{m}^{\text {hyd }}$ is the hydrodynamic couple per unit initial length exerted by the 
fluid on the filament due to anisotropic forces on a filament cross section; in particular, it specifically excludes the moment generated by the hydrodynamic force per unit initial length, $\boldsymbol{X} \times \boldsymbol{f}^{\text {hyd }}$, which has cancelled analogously to the above-mentioned $\boldsymbol{m}^{\text {ext }}$ term in the derivation of Eqn. (2.2).

One also needs to specify boundary conditions for the contact force and contact moment, the simplest of which are

$$
\boldsymbol{F}(0, t)=\boldsymbol{F}(L, t)=\boldsymbol{M}(0, t)=\boldsymbol{M}(L, t)=\mathbf{0},
$$

which correspond to force and torque free ends.

All contributions can be expanded using the triad of directors via

$$
\begin{gathered}
\boldsymbol{F}=\sum_{i=1}^{3} F^{i} \boldsymbol{D}^{i}, \boldsymbol{M}=\sum_{i=1}^{3} \boldsymbol{M}^{i} \boldsymbol{D}^{i}, \\
\boldsymbol{f}^{h y d}=\sum_{i=1}^{3} f^{h y d, i} \boldsymbol{D}^{i}, \boldsymbol{m}^{h y d}=\sum_{i=1}^{3} m^{h y d, i} \boldsymbol{D}^{i} .
\end{gathered}
$$

With no summation convention, the constitutive relations of the filament are given by (Lim et al., 2008)

$$
\begin{aligned}
F^{i} & =b_{i}\left(\boldsymbol{D}^{i} \cdot \frac{\partial \boldsymbol{X}}{\partial s}-\delta_{3 i}\right) \\
M^{i} & =a_{i} K_{i}
\end{aligned}
$$

with $K_{i}$ the generalised curvature vector so that the directors satisfy the Frenet-Serret formula,

$$
\frac{\partial \boldsymbol{D}^{i}}{\partial \eta}=\frac{\partial s}{\partial \eta} \boldsymbol{K} \times \boldsymbol{D}^{i}
$$

where $\eta$ denotes the physical arclength. In practice $\partial s / \partial \eta \approx 1$ for all time as the filament does not extend extensively for the parameter values used, reflecting that flagellar extension is beyond observational resolution in practice. This constitutive relation can also be derived by applying the variational principle to the following quadratic elastic energy (Lim et al., 2008),

$$
E=\frac{1}{2} \int_{0}^{L}\left[\sum_{i=1}^{3} a_{i} K_{i}^{2}+\sum_{i=1}^{3} b_{i}\left(\boldsymbol{D}^{i} \cdot \frac{\partial \boldsymbol{X}}{\partial s}-\delta_{3 i}\right)^{2}\right] \mathrm{d} s .
$$

Note therefore that the filament is straight in the absence of applied force and couples.

Returning to the local internal torques, which drive flagellar bending and swimming, we use

$$
\boldsymbol{m}^{i n t}=A_{1} s(L-s) \sin (k s-\omega t) \boldsymbol{D}^{1}+A_{2} s(L-s) \cos (k s-\omega t) \boldsymbol{D}^{2},
$$

where $A_{1}$ and $A_{2}$ govern the magnitude of the internal torque. In particular, this is modelled as an amplitude modulated travelling wave from proximal $(s=0)$ to distal (flagellar tail tip, $s=L)$ along the filament (Gaffney et al., 2011), with wavenumber $k$ and angular frequency $\omega=2 \pi / T$, where $T$ is the beat period.

In the presence of a wall, without loss of generality located at $z=\boldsymbol{e}_{3} \cdot \boldsymbol{X}=0$, we further consider a repulsive contact force when the filament is close to the wall, as motivated by the measurement of force between single cells and substrates (Klein et al., 2003; Sharp \& Dickinson, 2005). Hence, following 
numerous previous studies of sperm (Ishimoto \& Gaffney, 2015a, 2016) and more general microswimmers (Spagnolie \& Lauga, 2012) a short-range repulsive force is implemented and modelled via

$$
\boldsymbol{f}^{\text {ext }}=g \frac{e^{-z / d}}{1-e^{-z / d}} \boldsymbol{e}_{3}
$$

where $\boldsymbol{e}_{3}$ is the unit vector perpendicular to the wall (Fig. 2), with the parameters $g$ and $d$ representing the strength and decay lengthscale of the wall repulsive force, respectively.

\subsection{Elastohydrodynamic coupling}

For the small length and velocity scales associated with cellular swimming, the fluid flow will possess a negligible Reynolds number. Hence for a given forcing per unit volume $f^{b}$ Newtonian fluid dynamics is governed by Stokes' equations,

$$
\nabla p=\mu \Delta \boldsymbol{u}+\boldsymbol{f}^{b},
$$

where $p$ is the pressure field, the constant $\mu$ is fluid viscosity, and $f^{b}$ is the force exerted by the flagellar filament. The fluid forcing term arises due to the local force per unit initial length $-f^{\text {hyd }}$ and local torque per unit initial length

$$
-\left(\boldsymbol{X}(s, t) \times \boldsymbol{f}^{h y d}(\boldsymbol{X}(s, t))+\boldsymbol{m}^{h y d}(\boldsymbol{X}(s, t))\right)
$$

exerted on the fluid by the filament.

To proceed, consider the flow at field point $\boldsymbol{x}$ due to a regularised Stokeslet, of regularisation parameter $\varepsilon$ with free space decay boundary conditions and located at point $\boldsymbol{X}$, with a strength $\boldsymbol{f}$. This is denoted by $\boldsymbol{S}_{\varepsilon}[\boldsymbol{x}, \boldsymbol{X}, \boldsymbol{f}]$ with the analogous free space rotlet and potential dipole flows of strength $\boldsymbol{m}$ given by $\boldsymbol{R}_{\varepsilon}[\boldsymbol{x}, \boldsymbol{X}, \boldsymbol{m}]$ and $\boldsymbol{P}_{\varepsilon}^{D}[\boldsymbol{x}, \boldsymbol{X}, \boldsymbol{m}]$. Then, as detailed in the Appendices on the assumption of a circular filament cross section, the above force and torque profile generates the flow

$$
8 \pi \mu \boldsymbol{u}(\boldsymbol{x}, t)=-\int_{\Gamma}\left(\boldsymbol{S}_{\varepsilon}\left[\boldsymbol{x}, \boldsymbol{X}(s, t), \boldsymbol{f}^{h y d}(\boldsymbol{X}(s, t))\right]+\boldsymbol{R}_{\varepsilon}\left[\boldsymbol{x}, \boldsymbol{X}(s, t), \boldsymbol{m}^{h y d}(\boldsymbol{X}(s, t))\right]\right) d s .
$$

The vorticity is given by taking the curl of the above with respect to the field point $x$, and hence $\boldsymbol{w}$, which is defined as $\nabla \times \boldsymbol{u} / 2$ is given by

$$
8 \pi \mu \boldsymbol{w}(\boldsymbol{x}, t)=-\int_{\Gamma}\left(\boldsymbol{R}_{\varepsilon}\left[\boldsymbol{x}, \boldsymbol{X}(s, t), \boldsymbol{f}^{h y d}(\boldsymbol{X}(s, t))\right]+\frac{1}{4} \boldsymbol{P}_{\varepsilon}^{D}\left[\boldsymbol{x}, \boldsymbol{X}(s, t), \boldsymbol{m}^{h y d}(\boldsymbol{X}(s, t))\right]\right) d s,
$$

as also derived in the Appendices.

Furthermore, in the presence of a wall, the singularities in Eqn. (2.13) should be replaced by their associated wall-Stokeslet singularity, the Blakelet $\boldsymbol{S}_{\varepsilon}^{\text {wall }}$ and the wall-Rotlet $\boldsymbol{R}_{\varepsilon}^{\text {wall }}$, which are described in detail both in the Appendices and by Cortez \& Varela (2015). This yields

$$
8 \pi \mu \boldsymbol{u}(\boldsymbol{x}, t)=-\int_{\Gamma}\left(\boldsymbol{S}_{\varepsilon}^{\text {wall }}\left[\boldsymbol{x}, \boldsymbol{X}(s, t), \boldsymbol{f}^{\text {hyd }}(\boldsymbol{X}(s, t))\right]+\boldsymbol{R}_{\varepsilon}^{\text {wall }}\left[\boldsymbol{x}, \boldsymbol{X}(s, t), \boldsymbol{m}^{\text {hyd }}(\boldsymbol{X}(s, t))\right]\right) d s .
$$

Taking the curl of this expression with respect to the field point $x$ generates the vorticity field, and hence we have

$8 \pi \mu \boldsymbol{w}(\boldsymbol{x}, t)=-\frac{1}{2} \int_{\Gamma}\left(\nabla_{\boldsymbol{x}} \times \boldsymbol{S}_{\varepsilon}^{\text {wall }}\left[\boldsymbol{x}, \boldsymbol{X}(s, t), \boldsymbol{f}^{\text {hyd }}(\boldsymbol{X}(s, t))\right]+\nabla_{\boldsymbol{x}} \times \boldsymbol{R}_{\varepsilon}^{\text {wall }}\left[\boldsymbol{x}, \boldsymbol{X}(s, t), \boldsymbol{m}^{h y d}(\boldsymbol{X}(s, t))\right]\right) d s$. 
Note that the mirror image structure of the wall regularised Stokeslet and rotlet entail that their curl with respect to the field point cannot be expressed using a very small number of regularised singularities, though it is still tractable to calculate.

No-slip boundary conditions on the cell surface provide the additional constraints (Olson et al., 2013)

$$
\begin{aligned}
\frac{\partial \boldsymbol{X}}{\partial t}(s, t) & =\boldsymbol{u}(\boldsymbol{X}(s, t)), \\
\frac{\partial \boldsymbol{D}^{i}}{\partial t}(s, t) & =\boldsymbol{w}(\boldsymbol{X}(s, t), t) \times \boldsymbol{D}^{i}(s, t) .
\end{aligned}
$$

Finally note the filament is initially straight with no twist, so that all directors are constant as a function of arclength at initial time. This is sufficient to close the system, allowing a numerical algorithm for the coupled elastohydrodynamics of the filament, including the presence of a wall and a wall potential, as we now outline, closely following the structure laid down by Olson et al. (2013).

\section{Numerical Scheme}

Let time be discretised, with initial time $t_{0}$ and subsequent timepoints $t_{1}, t_{2}, \ldots$, while the flagellum centreline is discretised with $M$ immersed points with gridpoints at $s_{k}=\left(k-\frac{1}{2}\right) \Delta s$, for $k=1,2, \cdots, M$, where $\Delta s=L / M$.

Suppose the shape and director triad of the flagellum at time $t_{n}$ is known and consistent with the elastic boundary conditions. Then the contact force and moment, $\boldsymbol{F}, \boldsymbol{M}$ can be determined via the constitutive relations, Eqns. (2.6)-(2.7). In turn this allows the discretised approximations to the derivatives $\partial \boldsymbol{F} / \partial s, \partial \boldsymbol{M} / \partial s$ to be determined via central differences, using the elastic boundary conditions, Eqns 2.3 , to determine the derivatives at the boundary gridpoints. One subsequently can evaluate $f^{\text {hyd }}$ and $\boldsymbol{m}^{\text {hyd }}$ at the grid points along the filament via the force and moment balances, Eqns. (2.1)-(2.2). Now the velocity and vorticity vector fields at time $t_{n}$ can be determined via Eqns. (2.13)-(2.14), allowing a timestepping of the flagellum location and director triad via Eqns. (2.17)-(2.18) to timepoint $t_{n+1}$, allowing a time-marching of the flagellum dynamics.

\subsection{Timestepping, regularization and discretisation parameters}

The time-stepping in the above algorithm is explicit, conducted by the forward Euler method, where the timestep is taken to be $\Delta t=T / 80000$, noting $T=1 / 14$ seconds is the beat period, with further details documented in Olson et al. (2013). The grid-spacing along the flagellum and the regularization parameter are both given by $\varepsilon=\Delta s=L / 100$, where $L=56$ microns is the undeformed filament length, noting this is a typical human sperm flagellum length (Cummins \& Woodall, 1985). In particular, the regularization parameter, $\varepsilon$, represents the lengthscale over which the force extends for the regularized Dirac delta functions (e.g. Eqn (A.25) in the Appendices), and thus here is on the scale of the flagellum radius, for which a scale of $L / 100$ is appropriate for mammalian sperm, as detailed in the Supplementary material of Ishimoto \& Gaffney (2016). The numerical scheme parameters are summarized in Table 1 and the choice of the regularization parameter is documented in numerous studies (Smith, 2009; Gillies et al., 2009; Hyon et al., 2012), and we have followed the choices of Simons et al. (2015) whose filament elastohydrodynamic algorithm has been adapted for the present investigation. 
Table 1. Discretisation and regularization parameters used in the numerical scheme for modelling filament dynamics.

\begin{tabular}{|c|c|c|c|}
\hline Parameter & Interpretation & Value & Units \\
\hline \hline$\Delta s / L$ & flagellar discretisation & $1 / 100$ & - \\
\hline$\Delta t / T$ & time discretisation & $1 / 80000$ & - \\
\hline$\varepsilon / L$ & regularization parameter & $1 / 100$ & - \\
\hline
\end{tabular}

Table 2. A list of filament, wall potential and geometrical reference parameters used for simulating the filament dynamics. Note that the bending moduli and the twist modulus appear in the model only via the ratio of modulus to viscosity, with $\mu$ denoting viscosity, so the ratio is given, as discussed further in the text. Analogous comments apply for $b_{1}, b_{2}, b_{3}, A_{1}, A_{2}, g$.

\begin{tabular}{|c|c|c|c|}
\hline Parameter & Interpretation & Value & Units \\
\hline \hline$L$ & Initial filament length & 56 & $\mu \mathrm{m}$ \\
\hline$a_{1} / \mu$ & Bending modulus/Viscosity & $\{0.4,4\} \times 10^{4}$ & $\mu \mathrm{m}^{4} / \mathrm{s}$ \\
\hline$a_{2} / \mu$ & Bending modulus/Viscosity & $\{0.4,4,40\} \times 10^{4}$ & $\mu \mathrm{m}^{4} / \mathrm{s}$ \\
\hline$a_{3} / \mu$ & Twisting modulus/Viscosity & $\{0.4,4\} \times 10^{5}$ & $\mu \mathrm{m}^{4} / \mathrm{s}$ \\
\hline$b_{1} / \mu$ & Extensional modulus/Viscosity & $10^{6}$ & $\mu \mathrm{m}^{2} / \mathrm{s}$ \\
\hline$b_{2} / \mu$ & Extensional modulus/Viscosity & $b_{1} / \mu$ & $\mu \mathrm{m}^{2} / \mathrm{s}$ \\
\hline$b_{3} / \mu$ & Extensional modulus/Viscosity & $b_{1} / \mu$ & $\mu \mathrm{m}^{2} / \mathrm{s}$ \\
\hline$k L$ & Wave number & $4 \pi$ & - \\
\hline$T^{-1}$ & Beat frequency & 14 & $1 / \mathrm{s}$ \\
\hline$A_{1} / \mu$ & Torque Wave Amplitude/Viscosity & $\{1,3,4\} \times 10^{4}$ & $1 / \mathrm{s}$ \\
\hline$A_{2} / \mu$ & Torque Wave Amplitude/Viscosity & $\{0,0.2,0.6,1,2\} \times 10^{4}$ & $1 / \mathrm{s}$ \\
\hline$g T / \mu L$ & Wall repulsion strength & 50 & - \\
\hline$d$ & Wall repulsion length & 0.2 & $\mu \mathrm{m}$ \\
\hline
\end{tabular}

\subsection{Parameter Estimation}

Table 2 presents material and filament parameters with, as mentioned previously, the initial filament length, $L$, representing a typical human sperm (Cummins \& Woodall, 1985). Note the bending modulus $a_{1}$ and viscosity only appear in the model via the ratio $a_{1} / \mu$ as do the other mechanical parameters $a_{2}, a_{3}, b_{1}, b_{2}, b_{3}, g, A_{1}, A_{2}$ and hence we only specify these ratios in Table 2 .

Given the bending moment to viscosity ratio, one can determine the Sperm number, defined via

$$
S p:=\left(\frac{\xi L^{4}}{T a_{1}}\right)^{1 / 4}, \xi=\frac{4 \pi \mu}{\ln (2 L / r)},
$$

using the frequency $1 / T$ as the inverse timescale and $r=L / 100$ to represent the scale of the flagellum radius, with $\xi$ denoting a resistive force theory coefficient. While we do not use resistive force theory, its coefficient is incorporated in the definition of the Sperm number to enable comparison with previous studies as this number characterises the flexibility of the flagellum in theoretical filament mechanics.

In particular, for our typical reference parameter set below we have the Sperm number is 9.5. This is within the observed range of 2.8-17 generated by considering the relatively stiff sperm observed in mammals, of length 56 microns, swimming in media of viscosity ranging from watery medium to highly viscous analogues of physiological mucus used in experiments (Gadêlha et al., 2010). In addition, for swimming sperm with a more flexible flagellum, due to the absence of reinforcing accessory structures, together with a length and beat frequency of approximately 130 microns and $1 \mathrm{~Hz}$ respectively, which is 
typical of passerine birds such as pigeon (Vernon \& Woolley, 1999), the range of $S p$ is about 1.6-9.5. Hence, with $S p \approx 9.5$ we are considering a physiologically appropriate and relevant regime of bending moments, viscosities and frequencies. Alterations of the bending moduli such that $S p=16.7$ are also briefly considered below, thus examining the impact of swimming at higher Sperm number, where high curvature flagellum bends are observed to emerge (Smith et al., 2009b).

Further mechanical adjustments are considered, for example altering $a_{1} / \mu$ and $a_{2} / \mu$ so that the ratio $a_{2} / a_{1}$ is no longer unity to assess the impact of anisotropy. Furthermore, $a_{3} / \mu$ is sufficiently large to ensure that the filament does not readily twist as it deforms, as motivated by the fact that while twist might be indicated in detailed flagellar microscopy, it is not immediately apparent (Smith et al., 2009b). Finally, the stretch parameters, governed by $b_{1} / \mu, b_{2} / \mu, b_{3} / \mu$ are taken to be sufficiently large compared to bending moduli-viscosity ratios to ensure that any filament extension is below the resolution of current high-speed sperm microscopy given this does not reveal any clearly discernible stretching.

The wave number is not atypical for sperm (Smith et al., 2009b; Hong et al., 1993), while the torque wave amplitude is sufficient to ensure a suitable amplitude of the flagellum beat. Finally a wall repulsion lengthscale, $d$, is taken to be $200 \mathrm{~nm}$, slightly larger than the potential lengthscales observed by Klein et al. (2003) but details this close to the surface are qualitative only as the surface potential varies with cell, solute and surface, while they are not documented for sperm. The non-dimensional repulsion strength, $g T / \mu L$ is taken to be much larger than unity, so that repulsion is extensive once the cell is within hundreds of nanometres of the surface.

\section{Results: Swimming in free space}

We first consider the motion in free space, with no boundaries. At the initial time, the active filament representing a sperm flagellum is straight, so that at $t=0$ we have $\boldsymbol{X}=(-s, 0,-0)$, with $s \in[0, L]$, while the internal torque is given by Eqn. (2.10). In the initial simulations, we consider isotropic moments, $a_{1} / \mu=a_{2} / \mu=a_{3} / \mu=4 \times 10^{4} \mu \mathrm{m}^{4} / \mathrm{s}$, which give a Sperm number of $S p \approx 9.5$ and conduct numerical simulations for both planar and helical patterns of internal torque. The time evolution of the $x$ coordinate associated with the proximal end, $\boldsymbol{X}(s=0) \cdot \boldsymbol{e}_{x}$, is plotted in Fig. 3 for parameters of torque amplitude given by $A_{1} / \mu=3 \times 10^{4} / \mathrm{s}$, and $A_{2}=0$ for the planar case with $A_{2} / \mu=6 \times 10^{3} / \mathrm{s}$ for the helical case. As the simulation initiates, the proximal end point transiently reverses due to the filament bending induced by the internal torque, followed by a small positive swimming velocity, when the filament bends as a standing wave. As the filament subsequently begins to bend as a traveling wave, it starts to swim with nearly constant velocity. The swimming trajectories are essentially identical on comparing the helical and planar cases, which differ only by the value of $A_{2}$, which is non-zero for the helical case and generates local internal torques that induce non-planar beating. Such observations show that the additional internal torques associated with the $A_{2}$ contribution do not alter progressive motility but, instead, they induce an additional rotation of the sperm about its long axis that is observed in the simulations.

We proceed to consider reducing the bending moduli by an order of magnitude to $a_{1} / \mu=a_{2} / \mu=$ $4 \times 10^{3} \mu \mathrm{m}^{4} / \mathrm{s}$, and $a_{3} / \mu=4 \times 10^{5} \mu \mathrm{m}^{4} / \mathrm{s}$ which increases the Sperm number to $S p \approx 16.7$ with planar flagellar beating. In Fig. 4, snapshots of the predicted flagellar waveform are shown in terms of its projection onto the $x y$ plane at times $t / T \in\{0,1.5,3,4.5,6\}$, from left to right. Note though that the flagellum has been horizontally translated for ease of visualisation. At higher $S p$, the flagellum bends extensively, and thus attains high curvatures, as observed (Smith et al., 2009b), though one finds different 


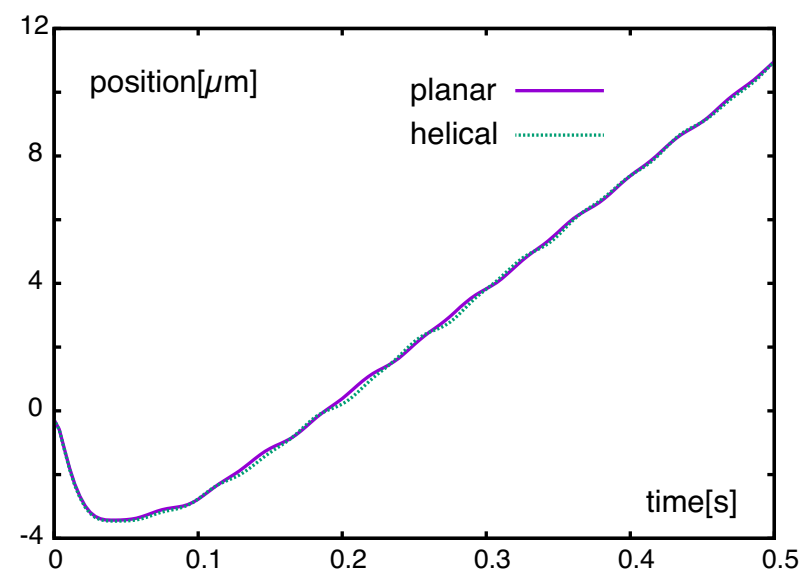

FIG. 3. The swimming distance in microns as a function of time for the model active filament representation of a sperm flagellum, with two- and three-dimensional internal torque generation in free space. The bending moduli are isotropic with $a_{1} / \mu=a_{2} / \mu=$ $a_{3} / \mu=4 \times 10^{4} \mu \mathrm{m}^{4} / \mathrm{s}$, and the torque generation parameters are given by $A_{1}=3 \times 10^{4} / \mathrm{s}$, and $A_{2}=0$ for the planar case, with $A_{2} / \mu=6 \times 10^{3} / \mathrm{s}$ for the helical case.

regions of the flagellum can approach within a filament radius of each other, which is the scale of the regularisation parameter in the simulations. Hence the numerics loses its validity for such high $S p$ dynamics, and we therefore focus on the previous case, $S p \approx 9.5$.

We subsequently alter the bending modulus to $a_{1} / \mu=4 \times 10^{3} \mu \mathrm{m}^{4} / \mathrm{s}$ and $a_{2}=a_{3}=4 \times 10^{5} \mu \mathrm{m}^{4} / \mathrm{s}$ which corresponds to an anisotropic filament, noting that many flagella are anisotropic due to their accessory structures. With isotropic internal torques governed by $A_{1} / \mu=A_{2} / \mu=1 \times 10^{4} / \mathrm{s}$, the resulting trajectory of the proximal end exhibits nearly open overlapping loops, when viewed from the swimming direction, as shown in Fig. 5(a). This trajectory projection becomes more flattened and nearly closed when instead the internal torque magnitude is anisotropic with $A_{1}=1 \times 10^{4} \mu \mathrm{m} / \mathrm{s}, A_{2} / \mu=$ $2 \times 10^{3} / \mathrm{s}$, and isotropic bending responses $a_{1} / \mu=a_{2} / \mu=4 \times 10^{4} \mu \mathrm{m}^{4} / \mathrm{s}$, with $a_{3} / \mu=4 \times 10^{5} \mu \mathrm{m}^{4} / \mathrm{s}$, as shown in Fig. 5(b). Once both the internal generation and bending moduli are anisotropic, the view of the trajectory in the direction of the swimming becomes much more anisotropic, Fig. 5(c). This is also analogous to the experimentally observed trajectory of a mammalian sperm head, viewed from the swimming direction, as depicted in Fig. 5(e) and also reproduced to good approximation in previous simulations with a prescribed ellipsoidal helical waveform (Fig. 5(d), from Smith et al. (2009a)). More generally, the results presented here suggest that the non-trivial looping behaviour of observed in mammalian sperm swimming requires anisotropy in both the flagellar structure and the generation of the internal torques, subject to the caveat that any sperm head mechanics is not incorporated in the modelling representation.

\section{Results: Swimming near a wall}

We now consider simulations in the presence of an infinite rigid wall at $z=0$. As found in the previous section, anisotropy of the bending modulus and torque generation are required for the trajectories of 


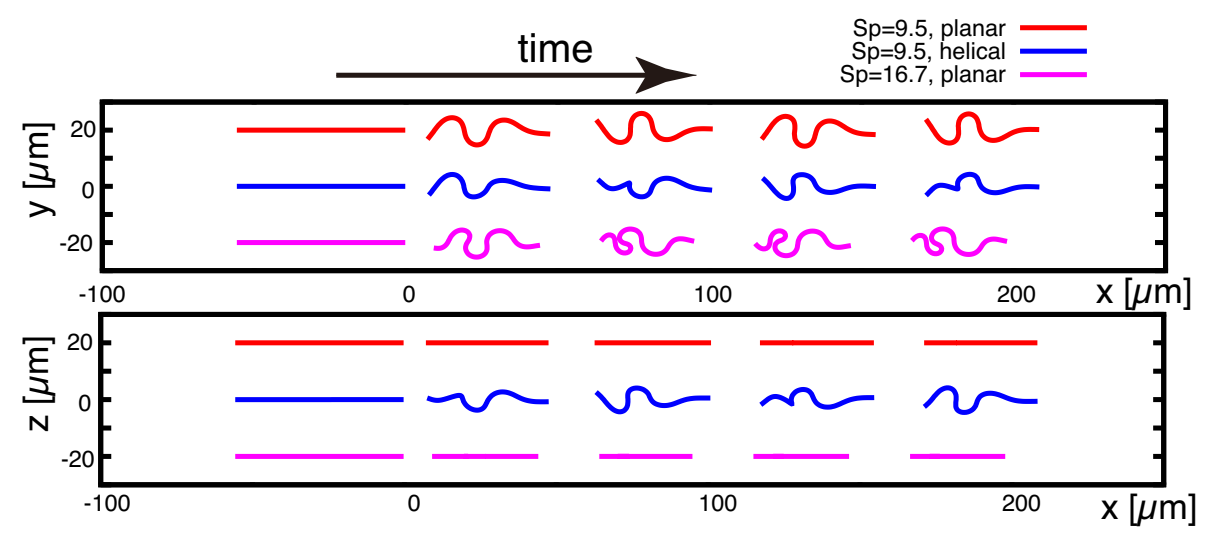

FIG. 4. Snapshots of the predicted filament shape projected onto the $x y$-plane (upper) and $x z$-plane (lower) for different times $t / T \in\{0,1.5,3,4.5,6\}$, with $t$ increasing from left to right. Note that the plots are translated horizontally for easy visualisation and the parameters are the same as in Fig. 3

the helical torque generators to resemble the observed looping of human sperm when viewed from the swimming direction. Hence, for helical torque generation, we restrict our attention to filaments with anisotropy in both the torques and moments throughout this section. In addition, initially the proximal filament end is located at $\boldsymbol{X}(s=0)=(0,0,10 \mu \mathrm{m})$, with the filament straight, oriented with $\boldsymbol{D}^{1} \cdot \boldsymbol{e}_{3}=0$ and pointing towards the wall with $\arccos \left(\boldsymbol{D}^{3} \cdot \boldsymbol{e}_{3}\right)=0.3 \pi$, so that the angle of attack is $\theta=0.2 \pi$. The resulting trajectory of the proximal end is plotted in Fig. 6.

Analogous to swimming in the absence of a wall, the virtual flagellum initially compresses, albeit very slightly, and reverses before eventually achieving steady swimming. When the proximal filament approaches the wall surface, it begins to rotate upwards due to the wall force. In the kinematic flagellum model, where the flagellum beat pattern is prescribed in the cell-fixed frame rather than emergent, the flagellar beat plane is typically nearly parallel to the wall (Ishimoto \& Gaffney, 2015a), as commonly observed (e.g. Smith et al. (2009b)). However, here with elastohydrodynamics and an emergent flagellar beat pattern, the wall repulsive force induces a rotation around the flagellar long axis, and the beat plane gradually becomes approximately vertical, perpendicular rather than parallel to the wall (Fig. 6). This hydrodynamic-induced rotation has also been found with no dynein contractions in the proximal flagellum, representing a filamentous head, as examined by turning off the internal couple for $s \in\left[0, s_{0}\right]$, $s_{0}=6.15 \mu \mathrm{m}$, in Eqn. (2.10) so that the inert sperm head is approximately $12 \%$ of cell length. The resulting perpendicular orientation is also be predicted by the kinematic model without wall repulsion though at relatively extreme parameter values and only for much greater wall-flagellum separation. The latter prediction of the kinematic model ensures such dynamics would be very easily disrupted as the wall-sperm interactions would be very weak at these separations (Ishimoto \& Gaffney, 2014). In contrast, here the predictions occur at small wall-sperm separations suggesting that at least one of the simplifications of the current model, such as the assumption of a filamentous head, is important in the dynamics of a planar beating sperm near a surface. 


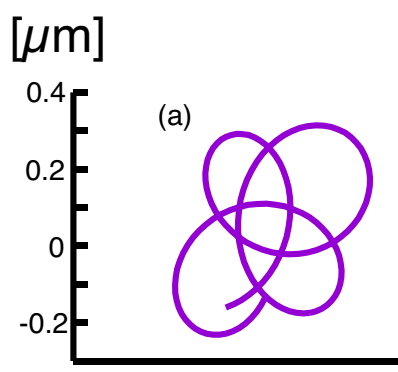

(d)

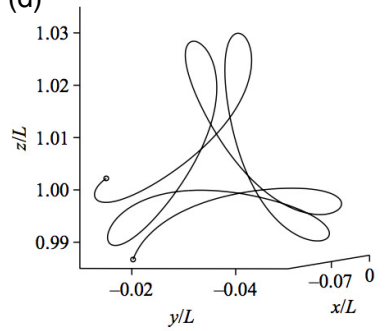

(b) modulus anisotropy torque anisotropy modulus/torque anisotropy

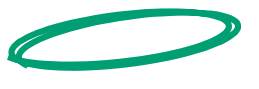

(c)

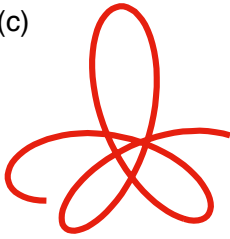

(e)

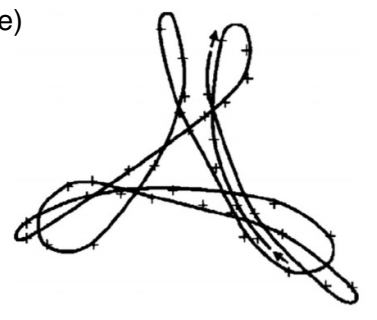

FIG. 5. Trajectories viewed from the swimming direction associated with a model filament for cases of isotropic and anisotropic bending moduli, combined with isotropic and anisotropic internal torque profiles. In particular we have the parameter values (a) $a_{1} / \mu=4 \times 10^{4} \mu \mathrm{m}^{4} / \mathrm{s}, a_{2} / \mu=a_{3} / \mu=4 \times 10^{5} \mu \mathrm{m}^{4} / \mathrm{s}$. $A_{1} / \mu=A_{2} / \mu=1 \times 10^{4} / \mathrm{s}$; (b) $a_{1} / \mu=a_{2} / \mu=a_{3} / \mu=4 \times 10^{4} \mu \mathrm{m}^{4} / \mathrm{s}$, $A_{1}=1 \times 10^{4} / \mathrm{s}, A_{2}=2 \times 10^{3} / \mathrm{s} ;$ (c) $a_{1} / \mu=4 \times 10^{4} \mu \mathrm{m}^{4} / \mathrm{s}, a_{2} / \mu=a_{3} / \mu=4 \times 10^{5} \mu \mathrm{m}^{4} / \mathrm{s}, A_{1} / \mu=1 \times 10^{4} / \mathrm{s}, A_{2} / \mu=2 \times 10^{3} / \mathrm{s}$. Further in plot (d) the trajectory for a model human view from the swimming direction is plotted via the hydrodynamic simulation of a human sperm with a prescribed ellipsoidal helical waveform, as reproduced from Smith et al. (2009a), with permission. Finally, in plot (e) the analogous plot is given for the observed mammalian sperm head trajectory projections, as presented in Woolley (2003).

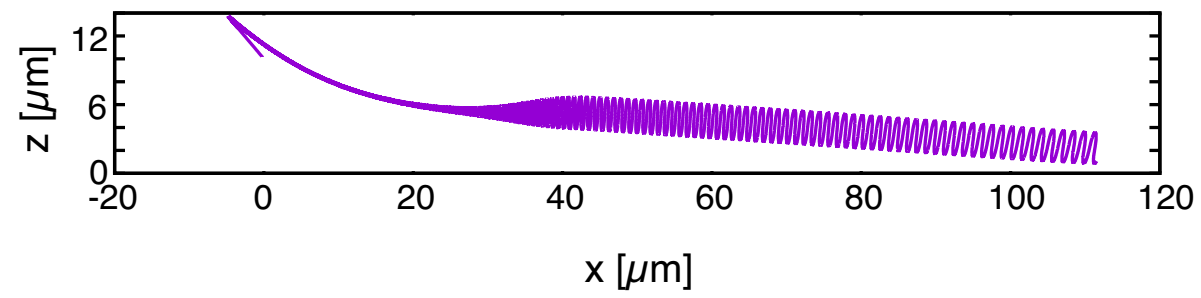

FIG. 6. Trajectory of the flagellar proximal end for the planar beat with anisotropic bending moduli, $a_{1} / \mu=4 \times 10^{4} \mu \mathrm{m}^{4} / \mathrm{s}$, $a_{2} / \mu=a_{3} / \mu=4 \times 10^{5} \mu \mathrm{m}^{4} / \mathrm{s}, A_{1} / \mu=4 \times 10^{4} / \mathrm{s}$, and $A_{2}=0$. The initial position of the proximal end is $(X, Y, Z)=(0,0,10 \mu \mathrm{m})$, and the initial angle of attack is given by $\theta=0.2 \pi$.

Further simulations have been conducted for helical torque generation. With the same initial conditions as the planar case, the projection of the virtual flagellum trajectory onto the wall, that is the $x y$-plane, is plotted in Fig. 7. Following an initial compression, the filament swims with nearly constant velocity, rotating about its long axis, and subsequently interacts with the wall. The flagellum then circles near the wall, and continues to rotate about its long axis, directly analogous to predictions of the kine- 

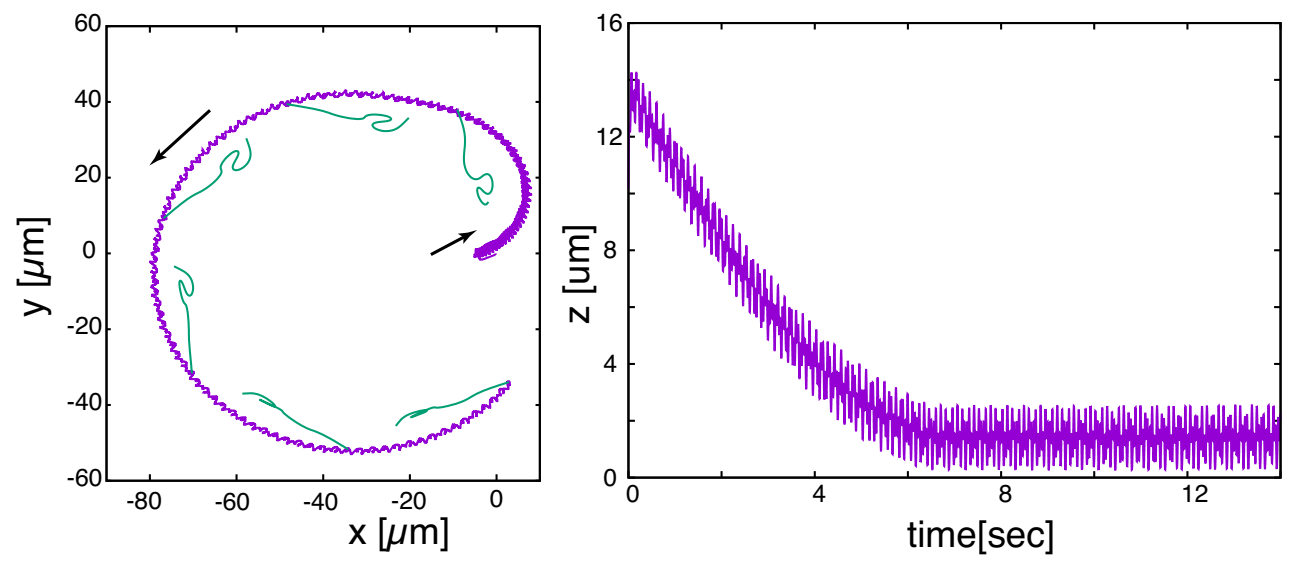

FIG. 7. (left) The trajectory of the flagellar proximal end for the helical beat, for a filament just above a no-slip boundary, with anisotropic bending moduli, $a_{1} / \mu=4 \times 10^{4} \mu \mathrm{m}^{4} / \mathrm{s}, a_{2} / \mu=a_{3} / \mu=4 \times 10^{5} \mu \mathrm{m}^{4} / \mathrm{s}$, and $A_{1} / \mu=4 \times 10^{4} / \mathrm{s}, A_{2}=2 \times 10^{4} / \mathrm{s}$. The arrows indicate the direction of swimming with increasing time. The initial positions are $(X, Y, Z)=(0,0,10 \mu \mathrm{m})$, with the initial angle of attack given by $\theta=0.2 \pi$. Snapshots of the flagellar waveform projected onto the $x y$-plane are also shown. Note that the anti-clockwise trajectory here contrasts with the clockwise pigeon sperm trajectory of in Fig 1 because of the different viewpoints: the filament is above the cover slip here while the sperm in below the coverslip in Fig 1. (right) The time evolution of the distance of the flagellar proximal end from the surface, $z$, for the same simulation presented in the left panel.

matic model, (Ishimoto \& Gaffney, 2015a, 2016), though the radius of the circling is much smaller than a kinematic flagellum (Ishimoto \& Gaffney, 2015a). The latter arises due to the extensive asymmetry in the emergent flagellar waveform with respect to the flagellar long axis, due to the flagellar elasticity. In Fig. 7, snapshots of the flagellar waveform are superimposed within the trajectory, highlighting the extensive asymmetry inducing the tight circling.

\section{Results: Swimming near a wall with a background shear flow}

We proceed to consider the behaviour of the active filament near a wall, in the presence of a background shear flow. In particular background flows often induce the reorientation of a swimmer to move upstream, which is a guidance cue known as (positive) rheotaxis, and observed in sperm, bacteria and artificial swimmers (Miki \& Clapham, 2013; Kantsler et al., 2014; Tung et al., 2015; Hill et al., 2007; Kaya \& Kose, 2012; Palacci et al., 2015). Furthermore, recent numerical and theoretical studies (Kantsler et al., 2014; Ishimoto \& Gaffney, 2015a) have successfully demonstrated that hydrodynamic torques drive the swimmer reorientation in the presence of a shear flow (Kantsler et al., 2014; Ishimoto \& Gaffney, 2015a; Ishimoto, 2017).

In Fig. 8, the trajectories of planar swimmers with the same parameters and initial conditions as those used in Fig. 6 are shown, but with the additional influence of a background linear shear flow, given by $\boldsymbol{u}^{\infty}=\dot{\gamma} z \boldsymbol{e}_{2}$, where $\dot{\gamma}$ is the constant shear strength and the cases of $\dot{\gamma}=0 / \mathrm{sec}, 0.1 / \mathrm{sec}$ and $1 / \mathrm{sec}$ are considered. As shown in the left panel of Fig. 8, the weak shear case $(\dot{\gamma}=0.1 / \mathrm{sec})$ shows a rheotactic response of the cell, whilst the strong shear $(\dot{\gamma}=1 / \mathrm{sec})$ flow sweeps the cell downstream. In the weak shear case, the cell still continuously retains proximity to the wall, as plotted in the right panel of Fig. 8. This contrasts the strong shear case, where the cell drifts from the near-wall region, and all the above results are consistent with observations (Kantsler et al., 2014) and kinematic flagellar swimming 

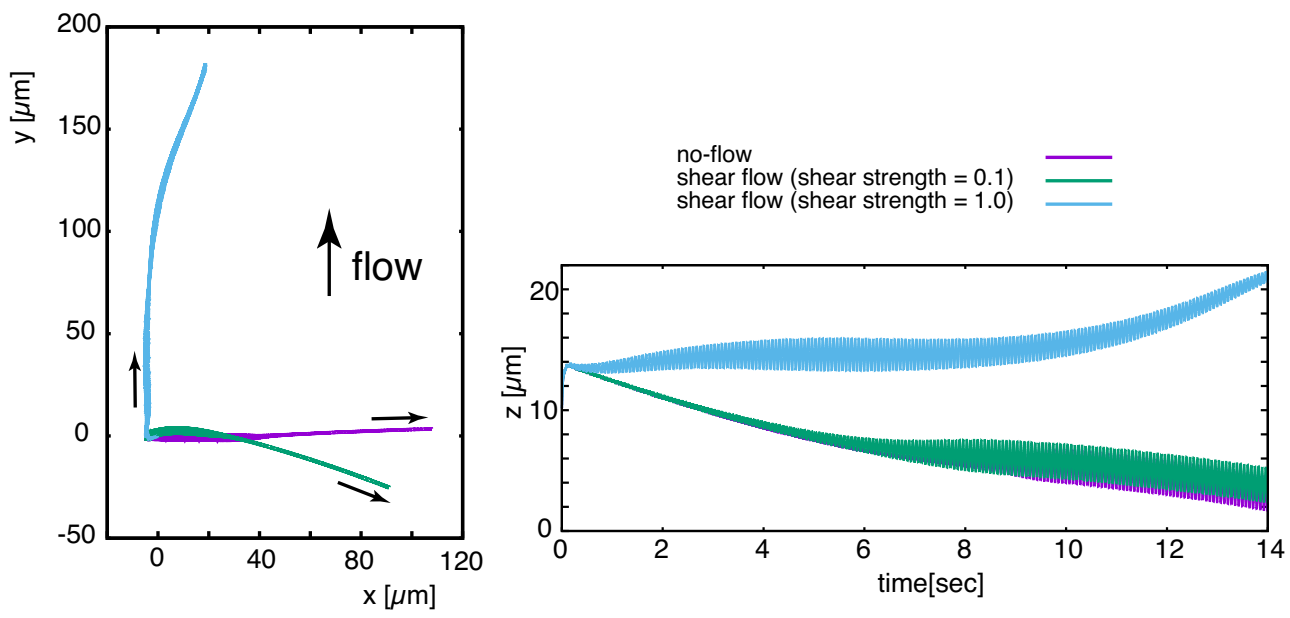

FIG. 8. (left) The trajectory of the active filament swimmer, as marked by the flagellar proximal end, given a planar internal torque generation in the presence of a background flow, with the arrows highlighting the direction of motion and the direction of the flow. The flagellar parameters and initial conditions are the same as those for Fig. 6 . The background shear flow is given by $\boldsymbol{u}^{\infty}=\dot{\gamma} z \boldsymbol{e}_{2}$, where the coordinate system is defined in Figure 2, and the shear strength is given by $\dot{\gamma}=0.1 / \mathrm{sec}$, and $1 / \mathrm{sec}$. (right) Time evolution of the height of the flagellar proximal end from the wall, $z$, in the same simulation as in the left panel.

simulations (Ishimoto \& Gaffney, 2015a) for planar beaters.

We proceed to consider whether a filamentous swimmer with a helical internal torque generation exhibits rheotaxis. The shear flow is once more given by $\boldsymbol{u}^{\infty}=\dot{\gamma} z \boldsymbol{e}_{2}$, now with $\dot{\gamma}= \pm 0.1 / \mathrm{sec}$. It can be clearly seen from Fig. 9 that there is no rheotaxis; in particular, the cell trajectory rotates in the $x-y$ plane, with the cell maintaining proximity to the wall regardless of the background shear flow, with no reorientation of the cell to swim upstream. Such observations are also in agreement with previous kinematic studies (Ishimoto \& Gaffney, 2015a). More generally and as with kinematic simulations, during rheotaxis flagellar proximity to the wall prevents the swimmer being washed away due to suppression of the flow arising from the no-slip condition at the wall. In addition, the cell swimming trajectory needs to be sufficiently straight in the absence of a flow for rheotaxis (Ishimoto \& Gaffney, 2015a), else the torques induced by background flow are sub-dominant to the torques driving swimmer turning in the absence of flow and the flow consequently has minimal effect on orientation.

\section{Discussions and conclusions}

In this paper, we have considered an elastohydrodynamical model for an active filament representation of a virtual sperm in a viscous medium, in the absence of inertia. While the model relies heavily on the elastohydrodynamical framework developed by Olson et al. (2013), there is no longer the concept of curvature control to enable our objective of exploring the consequences of amplitude modulated travelling waves of internal forcing along the filament, as motivating by observations of human sperm (Smith et al., 2009b; Gaffney et al., 2011). In addition, we have also introduced a solid boundary and a background flow, to examine their impact on elastohydrodynamic swimming models. However, we typically must either neglect the effect of sperm head mechanics or restrict our attention to sperm morphologies with filamentous heads, such as sturgeon, starling and pigeon. 


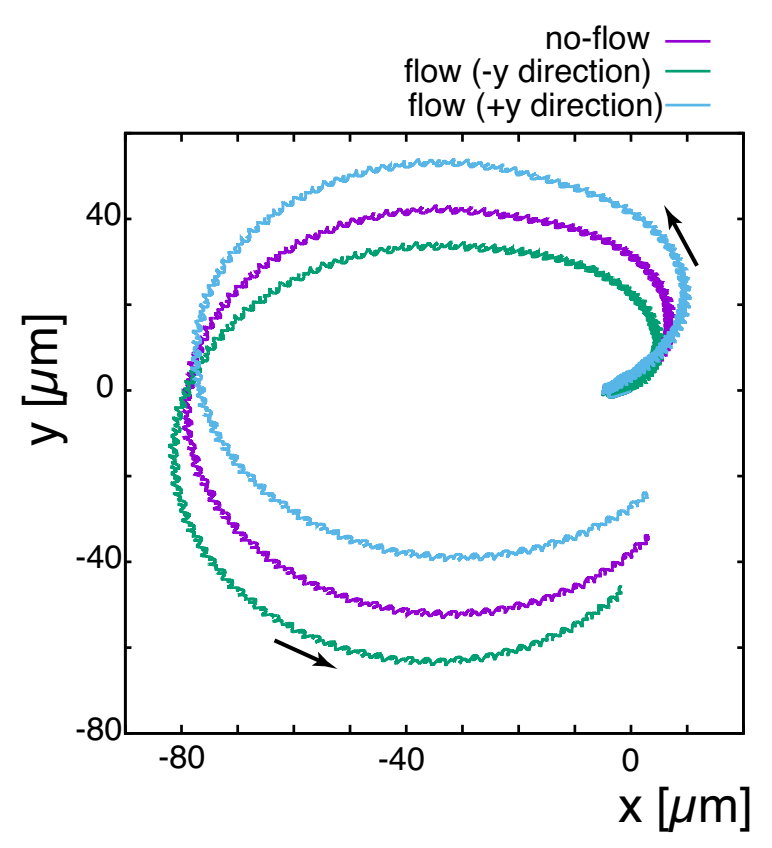

FIG. 9. Trajectory of the flagellar proximal end for filaments with helical internal torque generation in the presence of a background flow, with the arrows highlighting the direction of swimming. The flagellar parameters and initial conditions are the same as those for Fig. 7. The background shear flow is given by $\boldsymbol{u}^{\infty}=\dot{\gamma}_{z} \boldsymbol{e}_{2}$, where the coordinate system is defined in Figure 2, and the shear rate is given by $|\dot{\gamma}|=0.1 / \mathrm{sec}$ when shear is present.

In initial simulations, with the absence of a boundary, planar waveforms are generated for virtual flagella with axisymmetric elastic properties and internal moments, while helical beat patterns can be generated with simple helical waves of torque. Further simulations reinforce such observations and suggest that simple patterns of internal torque are sufficient to generate planar and 3D beating patterns that are qualitatively similar to those observed in practice for eukaryotic flagella, though the 3D waves do require both binormal and normal internal moments. In addition, subject to the caveat of neglecting sperm head mechanics, the modelling framework also suggests that the characteristic and non-trivial looping of the human sperm head when the swimming cell is viewed head on requires anisotropy in both the internal torque generation and the flagellum bending moduli.

Furthermore, the extensive variety of predicted waveforms has also reflected the diverse types of observed patterns, especially once anisotropic material parameters were considered, representing the diversity of accessory structures among sperm flagella (Baltz et al., 1990; Vernon \& Woolley, 1999; Psenicka et al., 2007). However, the prospect of establishing quantitative agreement is beyond the scope of this study and requires arc-length dependent estimates for material parameters. Such measurements are limited, with measurements of bending stiffness for select sperm, such as bull, rat and sea-urchin (Okuno \& Hiramoto, 1979; Schmitz-Leisch \& Lindemann, 2004; Leisch et al., 2008), while bending and shear moduli are available for the flagellated alga Chlamydomonas reinhardtii (Xu et al., 2016), though tensile stiffness has not been measured.

The absence of an inextensibility constraint has led to the absence of the buckling instability seen in inextensible flagellum models at higher Sperm number (Gadêlha et al., 2010). Nonetheless, the actual extension of the flagellum in the simulations is extremely small, less than 30 nanometres, which is 
undetectable in typical sperm microscopy studies, so that such marginal extensibility of the flagellum cannot be ruled out. Hence flagellar buckling instabilities need not be necessary with increasing Sperm number, and thus may not be a cause of high Sperm number features of flagellar beating, such as extreme curvatures and asymmetric beating (Woolley \& Vernon, 2001; Gadêlha et al., 2010).

On introducing a wall, stable boundary swimming was observed for virtual flagellar filaments with either helical and planar internal torques, together with the concomitant waveforms. For helical internal torques the virtual swimming flagella boundary accumulated with an emergent asymmetric waveform, which introduced tightly circling trajectories near the boundary. In particular, such behaviour of helical beating, rolling and close-surface circular trajectories has been observed for avian sperm with filamentous heads such as pigeon, starling and domestic fowl (Vernon \& Woolley, 1999), as well as other sperm more generally (Woolley, 2003). In studies with fixed flagellar waveforms, helical swimmers tended not to cycle so tightly near a surface and such circling sperm also did not undergo rheotaxis, that is reorient in response to a background flow (Ishimoto \& Gaffney, 2015a). It is clearly seen that the tight circling is retained with background flow for the current framework with its emergent flagellum beat pattern.

This firstly further supports the prediction that circling sperm do not rheotax, emphasising that flagellar elasticity is not fundamental in explaining why circling sperm do not exhibit a directional response to background flow. More generally the fact flagellar elasticity induces a tighter circling also indicates that kinematic models will overpredict the propensity of sperm to rheotax since the underprediction of circling entails an underprediction of the torques on the sperm, which in turn the influence of a background flow must counterbalance to induce rheotaxis.

As previously mentioned, a notable restriction in the modelling presented in this paper is the assumption of a filamentous morphology. Furthermore, the absence of the interaction between a non-filamentous head and boundary may be pertinent in why filaments with planar internal torques slowly rotated to become perpendicular to the boundary, in distinct contrast to common observation of planar beating sperm, such as mammalian sperm in high viscosity media (Smith et al., 2009b). While this is, at first inspection, a negative result it serves to highlight that flagellar elasticity can lead to unexpected results and thus it is vital to study in further detail, as well as indicating that the mechanics governing sperm behaviour near a surface is not fully understood. Such results further highlight that the current algorithm needs generalisation to consider general sperm head geometries. However, this also fully couples the elastic and fluid dynamics, and thus the simple implementation of the framework - its major advantage - is lost. Given further difficulties of numerical inefficiency and instability at higher and lower sperm numbers, there is a clear motivation for a reformulation of the current elastohydrodynamic algorithm in future work, which will also facilitate extensions such as considering the impact of a non-filamentous sperm head.

In summary, an elastohydrodynamic model of flagellum waveform formation, with travelling waves of internal torque has been explored for virtual sperm with a filamentous cell body or no head. The resulting waveforms are qualitatively similar to those observed in many sperm and emphasise that flagellar buckling instabilities need not occur at high sperm numbers. The study also supports the use of kinematic models of sperm swimming with fixed helical flagellar waveforms, in that flagellar elasticity does not disrupt the boundary accumulation dynamics of helical swimmers, though elasticity will reduce the tendency of a surface swimming virtual sperm to rheotax. Furthermore, the boundary accumulation studies presented also indicate that sperm head-boundary interactions are possibly required in fully reproducing subtle aspects of boundary accumulation for planar beating sperm swimmers in the context of elastohydrodynamics and highlight further directions for modelling development. This in turn is driven by the larger scale grand-challenge of integrating elastohydrodynamics with models of dynein regulation, to understand beat pattern emergence in flagella, and the analogous many body problem of 
cilia.

\section{Acknowledgements}

This work has been supported by JSPS-KAKENHI for young researchers (15H06314), Kyoto University Hakubi Project and Kyoto University Supporting Programme for Interaction-based Initiative Team Studies (SPIRITS). Elements of these simulations were performed using the cluster computing system within Research Institute for Mathematical Sciences (RIMS), and Institute for Information Management and Communication (IIMC), Kyoto University. K.I. acknowledges JSPS Overseas Research Fellowship for his stay in Mathematical Institute, University of Oxford. Further, EAG gratefully acknowledges Professor John R. Blake in stimulating and driving his entry into the fascinating field of flagellate, ciliate and ciliary dynamics.

\section{A. Regularized Stokeslets}

In this Appendix, we provide a comprehensive introduction to the derivations of core properties of regularized Stokes singularities, together with a collation of derivations for the results used in the simulation framework. The results are not novel and the reader is referred to the literature for the original papers (Cortez, 2001; Ainley et al., 2008; Simons et al., 2015; Cortez \& Varela, 2015).

\section{A.1 Regularized Stokes equations}

We consider the three-dimensional Stokes equation in the presence of an external force

$$
\nabla p=\mu \Delta \boldsymbol{u}+\boldsymbol{f}^{b} \text { with } \nabla \cdot \boldsymbol{u}=0
$$

where $f^{b}$ is the force per unit volume exerted on the fluid.

For the derivation of the regularized Stokeslets, we introduce a non-singular, spatially-localized, force $\boldsymbol{f}\left(\boldsymbol{x}, \boldsymbol{x}_{0}\right)$ via

$$
\boldsymbol{f}^{b}\left(\boldsymbol{x}, \boldsymbol{x}_{0}\right)=\boldsymbol{f}_{0}\left(\boldsymbol{x}_{0}\right) \psi_{\varepsilon}\left(\boldsymbol{x}, \boldsymbol{x}_{0}\right)+\frac{1}{2} \nabla \times\left(\boldsymbol{m}_{0}\left(\boldsymbol{x}_{0}\right) \psi_{\varepsilon}\left(\boldsymbol{x}, \boldsymbol{x}_{0}\right)\right) .
$$

Here $\boldsymbol{x}$ is the field point, $\boldsymbol{x}_{0}$ is the source point, that is the centroid of the singularity, and the differential operator $\nabla$, together with all differential operators below, are with respect to the field point $x$. The vectors $\boldsymbol{f}_{0}$ and $\boldsymbol{n}_{0}$ are respectively the strength of the force and torque and in a linear superposition of localized forces, these will possess a dependence on $x_{0}$, representing the strength of the localised force at the source point $\boldsymbol{x}_{0}$. Finally, $\psi_{\varepsilon}$ is the blob or cutoff function to regularize the Dirac delta function, which is radially-symmetric and converges to the delta function as $\varepsilon \rightarrow 0$ with

$$
\int_{\mathbb{R}^{3}} \psi_{\varepsilon}\left(\boldsymbol{x}, \boldsymbol{x}_{0}\right) d \boldsymbol{x}=1
$$

A suitable choice of this function is

$$
\psi_{\varepsilon}\left(x, x_{0}\right)=\frac{15 \varepsilon^{4}}{8 \pi\left(r^{2}+\varepsilon^{2}\right)^{7 / 2}}
$$

where $r=\left|\boldsymbol{x}-\boldsymbol{x}_{0}\right|$. 
Let us introduce a regularized scalar Green's function $G_{\varepsilon}\left(\boldsymbol{x}, \boldsymbol{x}_{0}\right)$ and a biharmonic function $B_{\varepsilon}\left(\boldsymbol{x}, \boldsymbol{x}_{0}\right)$ and following Cortez (2001), we impose

$$
\Delta G_{\varepsilon}\left(\boldsymbol{x}, x_{0}\right)=\psi_{\varepsilon}\left(x, x_{0}\right), \quad \Delta B_{\varepsilon}\left(x, x_{0}\right)=G_{\varepsilon}\left(x, x_{0}\right),
$$

where the derivatives implicit in the Laplacian are with respect to the field point $x$. For free space, with decay boundary conditions at infinity, the resulting translational invariance ensures that $G_{\varepsilon}\left(\boldsymbol{x}, \boldsymbol{x}_{0}\right)$, and $B_{\varepsilon}\left(\boldsymbol{x}, x_{0}\right)$, depend on $\boldsymbol{x}$ and $\boldsymbol{x}_{0}$ only via their difference $\boldsymbol{r}=\boldsymbol{x}-\boldsymbol{x}_{0}$, while spatial isotropy further restricts this dependence to be simply on $r=|\boldsymbol{r}|$. However this does not hold more generally, for example with wall boundary conditions, which break the spatial symmetries.

Taking the divergence of the forced Stokes equations gives

$$
\Delta p\left(\boldsymbol{x}, \boldsymbol{x}_{0}\right)=\boldsymbol{f}_{0}\left(\boldsymbol{x}_{0}\right) \cdot \nabla \psi_{\varepsilon}\left(\boldsymbol{x}, \boldsymbol{x}_{0}\right) .
$$

Hence from Eqn. (A.5) with free space boundary conditions and, without loss, $p$ decaying to zero at spatial infinity, we have

$$
p\left(x, x_{0}\right)=f_{0}\left(x_{0}\right) \cdot \nabla G_{\varepsilon}\left(x, x_{0}\right),
$$

using textbook uniqueness results, as there is no mathematical singularity for $\varepsilon>0$. Substituting this solution into Stokes equations yields

$$
\mu \Delta \boldsymbol{u}=\left(\boldsymbol{f}_{0}\left(\boldsymbol{x}_{0}\right) \cdot \nabla\right) \nabla G_{\varepsilon}\left(\boldsymbol{x}, \boldsymbol{x}_{0}\right)-\boldsymbol{f}_{0}\left(\boldsymbol{x}_{0}\right) \psi_{\varepsilon}\left(\boldsymbol{x}, \boldsymbol{x}_{0}\right)-\frac{1}{2} \nabla \times\left(\boldsymbol{m}_{0}\left(\boldsymbol{x}_{0}\right) \psi_{\varepsilon}\left(\boldsymbol{x}, \boldsymbol{x}_{0}\right)\right) .
$$

By the use of Eqns. (A.2), (A.5) we have the velocity vector

$$
\mu \boldsymbol{u}=\left(\boldsymbol{f}_{0}\left(\boldsymbol{x}_{0}\right) \cdot \nabla\right) \nabla B_{\varepsilon}\left(\boldsymbol{x}, \boldsymbol{x}_{0}\right)-\boldsymbol{f}_{0}\left(\boldsymbol{x}_{0}\right) G_{\varepsilon}\left(\boldsymbol{x}, \boldsymbol{x}_{0}\right)+\frac{1}{2} \boldsymbol{m}_{0}\left(\boldsymbol{x}_{0}\right) \times \nabla G_{\varepsilon}\left(\boldsymbol{x}, \boldsymbol{x}_{0}\right) .
$$

The velocity induced by a localised force is known as the regularized Stokeslet,

$$
\boldsymbol{S}_{\varepsilon}\left[\boldsymbol{x}, \boldsymbol{x}_{0}, \boldsymbol{f}_{0}\left(\boldsymbol{x}_{0}\right)\right]:=\left(\boldsymbol{f}_{0}\left(\boldsymbol{x}_{0}\right) \cdot \nabla\right) \nabla B_{\varepsilon}\left(\boldsymbol{x}, \boldsymbol{x}_{0}\right)-\boldsymbol{f}_{0}\left(\boldsymbol{x}_{0}\right) G_{\varepsilon}\left(\boldsymbol{x}, \boldsymbol{x}_{0}\right)
$$

Alternatively, we can rewrite the expression in the form,

$$
\boldsymbol{S}_{\varepsilon}\left[\boldsymbol{x}, \boldsymbol{x}_{0}, \boldsymbol{f}_{0}\left(\boldsymbol{x}_{0}\right)\right]=H_{1}(r) \boldsymbol{f}_{0}\left(\boldsymbol{x}_{0}\right)+H_{2}\left(\boldsymbol{f}_{0}\left(\boldsymbol{x}_{0}\right) \cdot \boldsymbol{r}\right) \boldsymbol{r},
$$

where $\boldsymbol{r}=\boldsymbol{x}-\boldsymbol{x}_{0}, r=|\boldsymbol{r}|$ and $H_{1}$ is given by

$$
H_{1}(r)=\frac{B_{\varepsilon}^{\prime}}{r}-G_{\varepsilon}
$$

where prime ' denotes differentiation with respect to $r$ and noting that $G_{\varepsilon}\left(\boldsymbol{x}, \boldsymbol{x}_{0}\right)=G_{\varepsilon}(r), B_{\varepsilon}\left(\boldsymbol{x}, x_{0}\right)=$ $B_{\varepsilon}(r)$ for free space boundary conditions due to spatial homogeneity and isotropy. Further, $H_{2}$ is given by

$$
H_{2}(r)=\frac{B_{\varepsilon}^{\prime \prime}}{r^{2}}-\frac{B_{\varepsilon}^{\prime}}{r^{3}}
$$

The velocity generated by the localised torque is called the regularized rotlet, given by

$$
\boldsymbol{R}_{\varepsilon}\left[\boldsymbol{x}, \boldsymbol{x}_{\mathbf{0}}, \boldsymbol{m}_{0}\left(\boldsymbol{x}_{0}\right)\right]:=\frac{1}{2} \boldsymbol{m}_{0}\left(\boldsymbol{x}_{0}\right) \times \nabla G_{\varepsilon}\left(\boldsymbol{x}, \boldsymbol{x}_{\mathbf{0}}\right)=\frac{Q(r)}{2}\left(\boldsymbol{m}_{0}\left(\boldsymbol{x}_{0}\right) \times \boldsymbol{r}\right),
$$


with

$$
Q(r)=\frac{G_{\varepsilon}^{\prime}(r)}{r} .
$$

Similarly, the viscosity multiplied by the local angular velocity of fluid is given by

$$
\mu \boldsymbol{w}=\frac{\mu}{2} \nabla \times \boldsymbol{u}=\frac{1}{2}\left(\boldsymbol{f}_{0}\left(\boldsymbol{x}_{0}\right) \times \nabla G_{\mathcal{\varepsilon}}\left(\boldsymbol{x}, \boldsymbol{x}_{0}\right)\right)+\frac{1}{4} \boldsymbol{m}_{0}\left(\boldsymbol{x}_{0}\right) \psi_{\varepsilon}\left(\boldsymbol{x}, \boldsymbol{x}_{0}\right)-\frac{1}{4}\left(\boldsymbol{m}_{0}\left(\boldsymbol{x}_{0}\right) \cdot \nabla\right) \nabla G_{\mathcal{\varepsilon}}\left(\boldsymbol{x}, \boldsymbol{x}_{0}\right) .
$$

\section{A.2 Regularized Stokes multiplets}

A.2.1 Stokeslet dipole The Stokeslet dipole is often defined by the source point directional derivative of the Stokeslet rank 2 tensor, $S_{i, \varepsilon}\left[\boldsymbol{x}, \boldsymbol{x}_{0}, \boldsymbol{e}_{j}\right]$, where $\boldsymbol{e}_{j}$ is the $j^{\text {th }}$ Cartesian basis vector and $i$ labels the $i^{t h}$ spatial component of $\boldsymbol{S}_{\varepsilon}$ (Pozrikidis, 1992). In free space one can equivalently develop multipole theory using the directional derivative with respect to the field point, $\boldsymbol{x}$, (Kim \& Karrila, 2005) as the dependence of $\psi_{\varepsilon}, G_{\varepsilon}, B_{\varepsilon}$ and the Stokeslet rank 2 tensor on the field and source points is only via $\boldsymbol{r}=\boldsymbol{x}-\boldsymbol{x}_{0}$. Below we adopt the latter approach, taking directional derivatives with respect to the field point noting that, in the presence of a wall, directional derivatives with respect to the field point no longer generate higher order multipoles, as there is no longer spatial homogeneity and isotropy, and we instead explicitly determine the image system.

With $\boldsymbol{e}$ denoting a unit vector and $\boldsymbol{f}^{b}\left(\boldsymbol{x}, \boldsymbol{x}_{0}\right)=\boldsymbol{f}_{0}\left(\boldsymbol{x}_{0}\right) \psi_{\varepsilon}\left(\boldsymbol{x}, \boldsymbol{x}_{0}\right)$ a localised force on the fluid, we have that the regularised Stokeslet dipole, defined by the directional derivative of the regularised Stokeslet, is given via

$$
\boldsymbol{S}_{\varepsilon}^{D}\left[\boldsymbol{x}, \boldsymbol{x}_{0}, \boldsymbol{f}_{0}\left(\boldsymbol{x}_{0}\right), \boldsymbol{e}\right]=(\boldsymbol{e} \cdot \nabla) \boldsymbol{S}_{\varepsilon}\left[\boldsymbol{x}, \boldsymbol{x}_{0}, \boldsymbol{f}_{0}\left(\boldsymbol{x}_{0}\right)\right]
$$

which can be written as

$$
\boldsymbol{S}_{\varepsilon}^{D}\left[\boldsymbol{x}, \boldsymbol{x}_{0}, \boldsymbol{f}_{0}\left(\boldsymbol{x}_{0}\right), \boldsymbol{e}\right]=(\boldsymbol{e} \cdot \nabla)\left(\boldsymbol{f}_{0}\left(\boldsymbol{x}_{0}\right) \cdot \nabla\right) \nabla B_{\varepsilon}\left(\boldsymbol{x}, \boldsymbol{x}_{0}\right)-\boldsymbol{f}_{0}\left(\boldsymbol{x}_{0}\right)(\boldsymbol{e} \cdot \nabla) G_{\varepsilon}\left(\boldsymbol{x}, \boldsymbol{x}_{0}\right),
$$

or, equivalently,

$\boldsymbol{S}_{\varepsilon}^{D}\left[\boldsymbol{x}, \boldsymbol{x}_{0}, \boldsymbol{f}_{0}\left(\boldsymbol{x}_{0}\right), \boldsymbol{e}\right]=H_{2}\left(\boldsymbol{f}_{0}\left(\boldsymbol{x}_{0}\right) \cdot \boldsymbol{e}\right) \boldsymbol{r}+H_{2}\left(\boldsymbol{f}_{0}\left(\boldsymbol{x}_{0}\right) \cdot \boldsymbol{r}\right) \boldsymbol{e}+\frac{H_{1}^{\prime}(r)}{r}(\boldsymbol{e} \cdot \boldsymbol{r}) \boldsymbol{f}_{0}\left(\boldsymbol{x}_{0}\right)+\frac{H_{2}^{\prime}(r)}{r}\left(\boldsymbol{f}_{0}\left(\boldsymbol{x}_{0}\right) \cdot \boldsymbol{r}\right)(\boldsymbol{e} \cdot \boldsymbol{r}) \boldsymbol{r}$

from Eqns. (A.11)-(A.13).

A.2.2 Rotlet. The anti-symmetrized contribution to the Stokeslet dipole corresponds to the rotlet introduced in the previous subsection. Let us introduce two unit vectors perpendicular to $\boldsymbol{m}, \boldsymbol{e}^{\perp}$ and $\boldsymbol{e}^{\perp \perp}$, with $\boldsymbol{e}^{\perp} \cdot \boldsymbol{e}^{\perp \perp}=0$ and $\boldsymbol{e}^{\perp} \times \boldsymbol{e}^{\perp \perp}=\boldsymbol{m} /|\boldsymbol{m}|$. Then combining two Stokeslet dipoles yields

$$
\begin{aligned}
\frac{|\boldsymbol{m}|}{2}\left(\boldsymbol{S}_{\varepsilon}^{D}\left[\boldsymbol{x}, x_{0}, e^{\perp}, e^{\perp \perp}\right]\right. & \left.-S_{\varepsilon}^{D}\left[\boldsymbol{x}, \boldsymbol{x}_{0}, \boldsymbol{e}^{\perp \perp}, e^{\perp}\right]\right)=\frac{|\boldsymbol{m}|}{2}\left(\boldsymbol{e}^{\perp \perp}\left(\boldsymbol{e}^{\perp} \cdot \nabla\right) G_{\varepsilon}\left(\boldsymbol{x}, \boldsymbol{x}_{0}\right)-\boldsymbol{e}^{\perp}\left(\boldsymbol{e}^{\perp \perp} \cdot \nabla\right) G_{\varepsilon}\left(\boldsymbol{x}, \boldsymbol{x}_{0}\right)\right) \\
= & \frac{|\boldsymbol{m}|}{2}\left(\boldsymbol{e}^{\perp} \times \boldsymbol{e}^{\perp \perp}\right) \times \nabla G_{\varepsilon}\left(\boldsymbol{x}, \boldsymbol{x}_{0}\right)=\frac{\boldsymbol{m}}{2} \times \nabla G_{\varepsilon}\left(\boldsymbol{x}, \boldsymbol{x}_{0}\right)=\boldsymbol{R}_{\varepsilon}\left[\boldsymbol{x}, \boldsymbol{x}_{\mathbf{0}}, \boldsymbol{m}\right]
\end{aligned}
$$

A.2.3 Potential dipole. Another important family of the fundamental solutions of the Stokes equation are those of potential flow, which are vorticity-free and thus characterised by no local rotation of the fluid, $\boldsymbol{w}=0$. Such solutions are required to satisfy the boundary conditions. In the case of fluid volume conserving systems, the lowest singular solution is the potential dipole, which can be obtained by taking 
a point source dipole (Pozrikidis, 1992) or, as here, by applying the negative Laplacian, $-\nabla^{2}$, to the Stokeslet to yield

$$
\boldsymbol{P}_{\varepsilon}^{D}\left[\boldsymbol{x}, \boldsymbol{x}_{0}, \boldsymbol{f}_{0}\left(\boldsymbol{x}_{0}\right)\right]=\boldsymbol{f}_{0}\left(\boldsymbol{x}_{0}\right) \psi_{\varepsilon}\left(\left(\boldsymbol{x}_{0}\right)\right)-\left(\boldsymbol{f}_{0}\left(\boldsymbol{x}_{0}\right) \cdot \nabla\right) \nabla G_{\varepsilon}\left(\boldsymbol{x}_{0}, \boldsymbol{x}\right) .
$$

On recollecting that $\psi_{\varepsilon}\left(\boldsymbol{x}, \boldsymbol{x}_{0}\right)=\psi_{\varepsilon}(r)$, this can be rewritten as

$$
\boldsymbol{P}_{\varepsilon}^{D}\left[\boldsymbol{x}, \boldsymbol{x}_{0}, \boldsymbol{f}_{0}\left(\boldsymbol{x}_{0}\right)\right]=D_{1}(r) \boldsymbol{f}_{0}\left(\boldsymbol{x}_{0}\right)+D_{2}(r)\left(\boldsymbol{f}_{0}\left(\boldsymbol{x}_{0}\right) \cdot \boldsymbol{r}\right) \boldsymbol{r},
$$

where $D_{1}$ and $D_{2}$ are respectively given by

$$
D_{1}(r)=\frac{G_{\varepsilon}^{\prime}}{r}-\psi_{\varepsilon} \text { and } D_{2}(r)=\frac{G_{\varepsilon}^{\prime \prime}}{r^{2}}-\frac{G_{\varepsilon}^{\prime}}{r^{3}}
$$

Note therefore that Eqn A.16 can be reduced to

$$
\mu \boldsymbol{w}=\boldsymbol{R}_{\varepsilon}\left[\boldsymbol{x}, x_{0}, f_{0}\left(x_{0}\right)\right]+\frac{1}{4} \boldsymbol{P}_{\varepsilon}^{D}\left[x, x_{0}, m_{0}\left(x_{0}\right)\right] .
$$

\section{A.3 Regularized Stokeslets in presence of a flat wall}

A.3.1 Regularized Blakelet. In presence of an infinite no-slip rigid wall, the Stokeslet is referred to as the Blakelet after Blake (1971) in the singular case. Ainley et al. (2008) has derived the regularized version of the Blakelet, introducing two different blob functions, as ultimately required to ensure the boundary condition at the wall is satisfied,

$$
\psi_{\varepsilon}\left(\boldsymbol{x}, \boldsymbol{x}_{0}\right)=\frac{15 \varepsilon^{4}}{8 \pi r_{\varepsilon}^{7}} \text { and } \phi_{\varepsilon}\left(\boldsymbol{x}, \boldsymbol{x}_{0}\right)=\frac{3 \varepsilon^{2}}{4 \pi r_{\varepsilon}^{5}},
$$

where $r_{\varepsilon}$ is defined by $r_{\varepsilon}=\sqrt{r^{2}+\varepsilon^{2}}$ and the force is localised around the source point $x_{0}$, which is separated from the rigid wall at $\boldsymbol{x} \cdot \boldsymbol{e}_{3}=0$ by $h=\boldsymbol{x}_{0} \cdot \boldsymbol{e}_{3}>0$. The image singularity is located at $\tilde{\boldsymbol{x}}_{0}=\boldsymbol{x}_{0}-2 h \boldsymbol{e}_{3}$ and we define $\tilde{\boldsymbol{f}}=2\left(\boldsymbol{f} \cdot \boldsymbol{e}_{3}\right) \boldsymbol{e}_{3}-\boldsymbol{f}$; then the regularized Blakelet is given by

$$
\begin{aligned}
\boldsymbol{S}_{\varepsilon}^{\text {wall }}\left[\boldsymbol{x}, \boldsymbol{x}_{0}, \boldsymbol{f}\right] & =\boldsymbol{S}_{\varepsilon}\left[\boldsymbol{x}, \boldsymbol{x}_{0}, \boldsymbol{f}\right]-\boldsymbol{S}_{\varepsilon}\left[\boldsymbol{x}, \tilde{\boldsymbol{x}}_{0}, \boldsymbol{f}\right] \\
& -h^{2} \boldsymbol{P}_{\varepsilon}^{D}\left[\boldsymbol{x}, \tilde{\boldsymbol{x}}_{0}, \tilde{\boldsymbol{f}}\right]+2 h \boldsymbol{S}_{\varepsilon}^{D}\left[\boldsymbol{x}, \tilde{\boldsymbol{x}}_{0}, \boldsymbol{e}_{3}, \tilde{\boldsymbol{f}}\right]+2 h \boldsymbol{R}_{\varepsilon}^{\psi}\left[\boldsymbol{x}, \tilde{\boldsymbol{x}}_{0}, \boldsymbol{f} \times \boldsymbol{e}_{3}\right]-2 h \boldsymbol{R}_{\varepsilon}^{\phi}\left[\boldsymbol{x}, \tilde{\boldsymbol{x}}_{0}, \boldsymbol{f} \times \boldsymbol{e}_{3}\right],
\end{aligned}
$$

where the Stokeslet and Stokeslet dipole terms are computed for the blob function $\psi_{\varepsilon}$, while in contrast the potential dipole term is determined using the blob function $\phi_{\varepsilon}$. The rotlet functions $\boldsymbol{R}_{\varepsilon}^{\psi}$ and $\boldsymbol{R}_{\varepsilon}^{\phi}$ are respectively determined using $\psi_{\varepsilon}$ and $\phi_{\varepsilon}$ in (A.25). The last two terms in (A.26) are derived from the difference between the two blob functions, and vanish as $\varepsilon \rightarrow 0$. We thus recover the singular Blakelet:

$$
\boldsymbol{S}_{0}^{\text {wall }}\left[\boldsymbol{x}, \boldsymbol{x}_{0}, \boldsymbol{f}\right]=\boldsymbol{S}_{0}\left[\boldsymbol{x}, \boldsymbol{x}_{0}, \boldsymbol{f}\right]-\boldsymbol{S}_{\varepsilon}\left[\boldsymbol{x}, \tilde{\boldsymbol{x}}_{0}, \boldsymbol{f}\right]-h^{2} \boldsymbol{P}_{0}^{D}\left[\boldsymbol{x}, \tilde{\boldsymbol{x}}_{0}, \tilde{\boldsymbol{f}}\right]+2 h \boldsymbol{S}_{\varepsilon}^{D}\left[\boldsymbol{x}, \tilde{\boldsymbol{x}}_{0}, \boldsymbol{e}_{3}, \tilde{\boldsymbol{f}}\right] .
$$

A.3.2 Regularized rotlet with an infinite flat wall. We proceed to consider the regularized version for the rotlet in presence of an infinite flat wall, which can be constructed in a similar manner to the regularized Blakelet (Ainley et al., 2008). The detailed derivation is given in Appendix B, and we just present the final expression,

$$
R_{\varepsilon}^{\text {wall }}[\boldsymbol{m}]=R_{\varepsilon}^{\phi}[\boldsymbol{m}]-\tilde{R}_{\varepsilon}^{\phi}[\boldsymbol{m}]+h \tilde{D}_{\varepsilon}^{P}[\boldsymbol{q}]-\tilde{S}_{\varepsilon}^{D}\left[\boldsymbol{q} ; \boldsymbol{e}_{3}\right]-\tilde{S}_{\varepsilon}^{D}\left[q \boldsymbol{e}_{3} ; \boldsymbol{q} / q\right]+\tilde{R}_{\varepsilon}^{\phi}\left[\boldsymbol{q}^{\perp}\right]-\tilde{R}_{\varepsilon}^{\psi}\left[\boldsymbol{q}^{\perp}\right]
$$

where $\boldsymbol{q}=\boldsymbol{m} \times \boldsymbol{e}_{3}$, and $\boldsymbol{q}^{\perp}=\boldsymbol{q} \times \boldsymbol{e}_{3}$ and $H_{1}, H_{2}$ are computed from the blob function $\psi_{\varepsilon}$, while $D_{1}, D_{2}$ are computed from $\phi_{\varepsilon}$. 


\section{A.4 Derivations for Section (2.2)}

Before considering regularised singularities, we first examine singular solutions with $\varepsilon=0$. As detailed in Pozrikidis (1992), a Stokeslet gives the flow associated with a point forcing and thus

$$
\boldsymbol{S}_{0}\left[\boldsymbol{x}, \boldsymbol{X}(s, t),-\boldsymbol{f}^{h y d}(\boldsymbol{X}(s, t))\right] d s=-\boldsymbol{S}_{0}\left[\boldsymbol{x}, \boldsymbol{X}(s, t), \boldsymbol{f}^{h y d}(\boldsymbol{X}(s, t))\right] d s
$$

generates the flow at the field point $\boldsymbol{x}$ associated with the hydrodynamic force per unit length, $-f^{h y d}$, on a fluid element of length $d s$ centred at a source point on the filament at Lagrangian coordinate $s$ and time $t$, i.e. $\boldsymbol{X}(s, t)$, with the equality immediate from linearity.

We also have a moment per unit length on the filament due to the fluid acting on the filament and thus, with a minus sign, the moment on the fluid by Newton's third law. As documented in Pozrikidis (1992) the only singularities that generate a couple are the Stokeslet, the Stokeslet dipole and the rotlet. The torque generated by the above Stokeslet precisely balances the torque per unit length, $-\boldsymbol{X}(s, t) \times$ $\boldsymbol{f}^{h y d}(\boldsymbol{X}(s, t))$, associated with the hydrodynamic force (Pozrikidis, 1992).

In principal either the Stokeslet dipole or the rotlet could generate a flow that would have the remaining required moment per unit length on the fluid $-\boldsymbol{m}^{\text {hyd }}$. If the Stokeslet dipole strength, which is a rank 2 tensor, is antisymmetric the resulting flow is equivalent to that of a rotlet. Otherwise, the symmetric contribution to the Stokeslet dipole strength generates a Stresslet singularity straining flow that is volume conserving but locally would act to distort a flagellum circular cross section. In contrast the rotlet flow locally acts to rotate, rather than distort, a circular cross section filament.

Noting the flagellum cross section is generally approximately circular we are motivated by the above observations to assume a rotlet should be used to generate the local couple. Thus the associated flow at the field point $\boldsymbol{x}$ due to the couple per unit length, $\left.-\boldsymbol{m}^{\text {hyd }} X(s, t)\right)$, on an element of length $d s$ centred at the source point on the filament $\boldsymbol{X}(s, t)$, is given by

$$
-\boldsymbol{R}_{0}\left[\boldsymbol{x}, \boldsymbol{X}(s, t), \boldsymbol{m}^{h y d}(\boldsymbol{X}(s, t))\right] d s .
$$

Approximating the singularities via their regularised counterparts with regularisation parameter $\varepsilon$, and invoking linearity to take a linear superposition generates Eqn. (2.13) of Section (2.2). Further, applying linear superposition to $\mu(\nabla \times \boldsymbol{u}) / 2=\mu \boldsymbol{w}$, noting Eqns (A.9), (A.16), (A.24) and (2.13), thus generates Eqn. (2.14) of Section (2.2).

When a wall is present at $\boldsymbol{x} \cdot \boldsymbol{e}_{3}=0$, we can simply replace the Stokeslet and Rotlet above with the Wall-Stokeslet and the Wall-rotlet to generate Eqn. (2.15) of Section (2.2). Taking half the curl of this expression with respect to the field point can be used to generate $\boldsymbol{w}$ in the presence of wall, though the final expression is not readily expressed or interpreted via a small number of regularised singularities, due to the extensive image singularities under consideration.

\section{Appendix B: The regularized rotlet in the presence of an infinite plane wall at $e_{3} \cdot x=0$.}

The derivation of the regularized image rotlet is provided, albeit in a more systematic way to Cortez \& Varela (2015). Let us consider the difference of the rotlet and its naive image on the wall at $\boldsymbol{e}_{3} \cdot \boldsymbol{x}=0$ to construct the image system, as in the singular case (Blake \& Chwang, 1974); in particular we have

$$
\boldsymbol{R}_{\varepsilon}\left[\boldsymbol{x}_{w}, \boldsymbol{x}_{0}, \boldsymbol{m}\right]-\boldsymbol{R}_{\varepsilon}\left[\boldsymbol{x}, \tilde{\boldsymbol{x}}_{0}, \boldsymbol{m}\right]=-\frac{1}{2} \frac{G_{\varepsilon}^{\prime}}{r} \boldsymbol{m} \times\left(\boldsymbol{x}_{0}-\tilde{\boldsymbol{x}}_{0}\right)=\frac{h G_{\varepsilon}^{\prime}}{r} \boldsymbol{e}_{3} \times \boldsymbol{m} .
$$

Hence the component perpendicular component to the wall satisfies the boundary condition. For the parallel components, we further add the regularized Stokeslets as in the singular case for the parallel 
vector $\boldsymbol{q}$, where $\boldsymbol{q} \cdot \boldsymbol{e}_{3}=0$ and we denote $q=|\boldsymbol{q}|$,

$$
h \boldsymbol{D}_{\varepsilon}^{P}\left[\boldsymbol{x}, \tilde{\boldsymbol{x}}_{0}, \boldsymbol{q}\right]-\boldsymbol{S}_{\varepsilon}^{D}\left[\boldsymbol{x}, \tilde{\boldsymbol{x}}_{0}, \boldsymbol{q}, \boldsymbol{e}_{3}\right]-\boldsymbol{S}_{\varepsilon}^{D}\left[\boldsymbol{x}, \tilde{\boldsymbol{x}}_{0}, q \boldsymbol{e}_{3}, \boldsymbol{q} / q\right],
$$

which can be evaluated at a field point on the wall, $\boldsymbol{x}_{w}$, from eq. (A.11), (A.19) and (A.22) to give

$$
h\left[D_{1}-\left(\frac{H_{1}^{\prime}}{r}+H_{2}\right)\right] \boldsymbol{q}+h\left(D_{2}-2 \frac{H_{2}^{\prime}}{r}\right)(\boldsymbol{q} \cdot \tilde{\boldsymbol{r}}) \tilde{\boldsymbol{r}}-\left(H_{2}+\frac{H_{1}^{\prime}}{r}\right)(\boldsymbol{q} \cdot \tilde{\boldsymbol{r}}) \boldsymbol{e}_{3},
$$

where $\tilde{\boldsymbol{r}}=\boldsymbol{x}_{w}-\tilde{\boldsymbol{x}}_{0}$

We can confirm that these terms can cancel the remaining velocity in Eqn. (A.29). The Green's function and the biharmonic function in the singular case or as $\varepsilon \rightarrow 0$ become

$$
G_{0}=-\frac{1}{4 \pi r}, \text { and } B_{0}=-\frac{r}{4 \pi}
$$

respectively, which yields

$$
D_{2}-2 \frac{H_{2}^{\prime}}{r}=H_{2}+\frac{H_{1}^{\prime}}{r}=0 .
$$

Therefore the combination of eq. (A.29) and (A.30) on the wall is reduced to

$$
\frac{h}{4 \pi r^{3}}\left(\boldsymbol{e}_{3} \times \boldsymbol{m}+\boldsymbol{q}\right) .
$$

Taking $\boldsymbol{q}=\boldsymbol{m} \times \boldsymbol{e}_{3}$ thus completes the boundary condition.

This cancellation does not occur in the regularized case as in the regularized Blakelet. Nonetheless, we can establish the image system by adding extra image rotlet terms as in the regularized Blakelet (Ainley et al., 2008). Only a single blob function of eq. (A.25) does not cancel the velocity induced by a regularized external force, but cancellation becomes possible using a combination of different blob functions. As in Ainley et al. (2008), the functions $H_{1}$ and $H_{2}$ are determined using $\psi_{\varepsilon}$, while $D_{1}$ and $D_{2}$ are derived from $\phi_{\varepsilon}$, yielding

$$
\begin{aligned}
& H_{1}=\frac{r^{2}+2 \varepsilon^{2}}{8 \pi r_{\varepsilon}^{3}}, H_{2}=\frac{1}{8 \pi r_{\varepsilon}^{3}}, Q^{\psi}=\frac{2 r^{2}+5 \varepsilon^{2}}{8 \pi r_{\varepsilon}^{5}} \\
& D_{1}=\frac{r^{2}-2 \varepsilon^{2}}{4 \pi r_{\varepsilon}^{5}}, D_{2}=\frac{-3}{4 \pi r_{\varepsilon}^{5}}, Q^{\phi}=\frac{1}{4 \pi r_{\varepsilon}^{3}} .
\end{aligned}
$$

Together with Proposition 1 of Ainley et al.,

$$
\frac{H_{2}^{\prime}}{r}-\frac{1}{2} D_{2}=0 \text { and } H_{2}-\frac{1}{2} D_{1}=-\frac{H_{1}^{\prime}}{r}-H_{2},
$$

we can reduce eq. (A.31) into

$$
h\left(\frac{2 r^{2}-\varepsilon^{2}}{8 \pi r_{\varepsilon}^{5}}\right) \boldsymbol{q}+\frac{3 \varepsilon^{2}}{8 \pi r_{\varepsilon}^{5}}\left(\boldsymbol{q} \cdot \tilde{\boldsymbol{x}}_{0}\right) \boldsymbol{e}_{3}
$$


As the rotlet induced by the blob function $\phi$ is used for $Q$, i.e. $Q^{\phi}$ in eq. (A.36), the combination of (A.38) and (A.29) will generate

$$
\begin{aligned}
& R_{\varepsilon}[\boldsymbol{m}]-\tilde{R}_{\varepsilon}[\boldsymbol{m}]+h \tilde{D}_{\varepsilon}^{P}[\boldsymbol{q}]-\tilde{S}_{\varepsilon}^{D}\left[\boldsymbol{q} ; \boldsymbol{e}_{3}\right]-\tilde{S}_{\varepsilon}^{D}\left[q \boldsymbol{e}_{3} ; \boldsymbol{q} / q\right] \\
= & \frac{3 \varepsilon^{2}}{8 \pi r_{\varepsilon}^{5}}\left[-\left(\boldsymbol{e}_{3} \cdot \tilde{\boldsymbol{x}}_{0}\right) \boldsymbol{q}+\left(\boldsymbol{q} \cdot \tilde{\boldsymbol{x}}_{0}\right) \boldsymbol{e}_{3}\right]=\left(Q^{\psi}-Q^{\phi}\right)\left(\boldsymbol{q} \times \boldsymbol{e}_{3}\right) \times \tilde{\boldsymbol{x}}_{0},
\end{aligned}
$$

by taking $\boldsymbol{q}=\boldsymbol{m} \times \boldsymbol{e}_{3}$. This in turn exactly corresponds to the difference of the rotlets produced by the two different blob functions,

$$
\tilde{R}_{\varepsilon}^{\psi}\left[\boldsymbol{q} \times \boldsymbol{e}_{3}\right]-\tilde{R}_{\varepsilon}^{\phi}\left[\boldsymbol{q} \times \boldsymbol{e}_{3}\right] .
$$

We therefore finally obtain the regularized rotlet expression in presence of an infinite rigid wall,

$$
R_{\varepsilon}^{\text {wall }}[\boldsymbol{m}]=R_{\varepsilon}^{\phi}[\boldsymbol{m}]-\tilde{R}_{\varepsilon}^{\phi}[\boldsymbol{m}]+h \tilde{D}_{\varepsilon}^{P}[\boldsymbol{q}]-\tilde{S}_{\varepsilon}^{D}\left[\boldsymbol{q} ; \boldsymbol{e}_{3}\right]-\tilde{S}_{\varepsilon}^{D}\left[q \boldsymbol{e}_{3} ; \boldsymbol{q} / q\right]+\tilde{R}_{\varepsilon}^{\phi}\left[\boldsymbol{q}^{\perp}\right]-\tilde{R}_{\varepsilon}^{\psi}\left[\boldsymbol{q}^{\perp}\right],
$$

where $\boldsymbol{q}=\boldsymbol{m} \times \boldsymbol{e}_{3}$, and $\boldsymbol{q}^{\perp}=\boldsymbol{q} \times \boldsymbol{e}_{3}$. The singular rotlet expression is straightforward to recover in the limit $\varepsilon \rightarrow 0$.

\section{REFERENCES}

Ainley, J., Durkin, S., Embid, R., Boindala, P. \& Cortez, R. (2008) The method of images for regularized Stokeslets. Journal of Computational Physics, 227, 4600-4616.

Baltz, J. M., Williams, P. O. \& Cone, R. A. (1990) Dense Fibers Protect Mammalian Sperm against Damage. Biology of Reproduction, 43, 485-491.

Bayly, P. V. \& Wilson, K. S. (2014) Equations of Interdoublet Separation during Flagella Motion Reveal Mechanisms of Wave Propagation and Instability. Biophysical Journal, 107, 1756-1772.

Bayly, P. V. \& Wilson, K. S. (2015) Analysis of unstable modes distinguishes mathematical models of flagellar motion. Journal of the Royal Society Interface, 12, 20150124.

Blake, J. R. (1971) A note on the image system for a stokeslet in a no slip boundary. Proceedings of Cambridge Philosophical Society, 70, 303-310.

Blake, J. R. \& Chwang, A. T. (1974) Fundamental singularities of viscous flow. Journal of Engineering Mathematics, 8, 23-29.

Brokaw, C. J. (1971) Bend Propagation By A Sliding Filament Model For Flagella. Journal of Experimental Biology, 55, 289-304.

Brokaw, C. J. (1972) Flagellar Movement: A Sliding Filament Model. Science, 178, 455-462.

Brokaw, C. J. (2002) Computer simulation of flagellar movement VIII: Coordination of dynein by local curvature control can generate helical bending waves. Cell Motility Cytoskeleton, 53, 103-124.

Brokaw, C. J. (2009) Simulation of cyclic dynein-driven sliding, splitting, and reassociation in an outer doublet pair. Biophysical Journal, 97, 2939-2947.

Camalet, S. \& Julicher, F. (2000) Generic aspects of axonemal beating. New Journal of Physics, 2, 24.1-24.2.

Cortez, R. (2001) The method of regularized Stokeslets. SIAM Journal on Scientific Computing, 23, 1204-1225.

Cortez, R. \& Varela, D. (2015) A general system of images for regularized Stokeslets and other elements near a plane wall. Journal of Computational Physics, 285, 41-54.

Coy, H. \& Gadêlha, H. (2017) Counterbend dynamics of cross-linked filament bundles and flagella. Journal of the Royal Society Interface, 14, 20170065.

Cummins, J. M. \& Woodall, P. F. (1985) On mammalian sperm dimensions. J. Reprod. Fert., 75, 153-175.

Dillon, R., Fauci, L. \& Yang, X. (2006) Sperm Motility and Multiciliary Beating: An Integrative Mechanical Model. Computers \& Mathematics with Applications, 52(5), 749 - 758.

Elgeti, J., Kaupp, U. \& Gompper, G. (2010) Hydrodynamics of sperm cells near surfaces. Biophysical Journal, 99, 1018-1026. 
Fauci, L. \& McDonald, A. (1995) Sperm motility in the presence of the boundaries. Bulletin of Mathematical Biology, 57, 679-699.

Fawcett, D. W. (1975) The Mammalian Sperm. Developmental Biology, 44, 394-436.

Fu, H. C., Wolgemuth, C. W. \& Powers, T. R. (2008) Beating patterns of filaments in viscoelastic fluids. Phys. Rev. $E, \mathbf{7 8}, 041913$.

Gadêlha, H., Gaffney, E. A., Smith, D. J. \& Kirkman-Brown, J. C. (2010) Non-linear instability in flagellar dynamics: a novel modulation mechanism in sperm migration?. Journal of the Royal Society Interface, 7, 1689-1697.

Gaffney, E., Gadêlha, H., Smith, D., Blake, J. \& Kirkman-Brown, J. (2011) Mammalian sperm motility: observation and theory. Annual Review of Fluid Mechanics, 43, 501-528.

Gillies, E. A., Cannon, R. M., Green, R. B. \& Pacey, A. A. (2009) Hydrodynamic propulsion of human sperm. Journal of Fluid Mechanics, 625, 445-474.

Gray, J. \& Hancock, G. (1955) The propulsion of sea-urchin spermatozoa. The Journal of Experimental Biology, 32, 802-814.

Hill, J., Kalkanci, O., McMurry, J. L. \& Koser, H. (2007) Hydrodynamic surface interactions enable it Escherichia Coli to seek efficient routes to swim upstream. Physical Review Letters, 98, 068101.

Hong, C. Y., Lee, M. F., Ou, M. C. \& Chao, H. T. (1993) Tail Beat Frequency of Human Sperm: Evaluated with Sperm Head Fixation Method and Computer-Assisted Semen Analysis. Archives of Andrology, 30(3), 171176.

Hyon, Y., Marcos, Powers, T. R., Stocker, R. \& Fu, H. C. (2012) The wiggling trajectories of bacteria. Journal of Fluid Mechanics, 705, 58-76.

Ishijima, S., Hamaguchi, M. S., Naruse, M., Ishijima, S. A. \& Hamaguchi, Y. (1992) Rotational movement of a spermatozoon around its long axis. Journal of Experimental Biology, 163, 15-31.

Ishimoto, K. (2017) Guidance of microswimmers by wall and flow: Thigmotaxis and rheotaxis of unsteady squirmers in two and three dimensions. Physical Review E, 96, 043101.

Ishimoto, K. \& Gaffney, E. A. (2014) A study of spermatozoan swimming stability near a surface. Journal of Theoretical Biology, 360, 187-199.

Ishimoto, K. \& Gaffney, E. A. (2015a) Fluid flow and sperm guidance: a simulation study of hydrodynamic sperm rheotaxis. Journal of the Royal Society Interface, 12, 20150172.

Ishimoto, K. \& Gaffney, E. A. (2015b) Modelling Spermatozoan Swimming: Its Capabilities and Limitations for Contributing to The Understanding of Sperm Guidance. In Cosson, J., editor, Flagellar mechanics and sperm guidance, page Chapter 9. Bentham Sciences.

Ishimoto, K. \& Gaffney, E. A. (2016) Mechanical tuning of mammalian sperm behaviour by hyperactivation, rheology and substrate adhesion: a numerical exploration. Journal of the Royal Society Interface, 13, 20160633.

Johnson, R. E. \& Brokaw, C. J. (1979) Flagellar Hydrodynamics: A Comparison Between Resistive-Force Theory And Slender-Body Theory. Biophysical Journal, 25, 113-127.

Kantsler, V., Dunkel, J., Blayney, M. \& Goldstein, R. E. (2014) Rheotaxis facilitates upstream navigation of mammalian sperm cells. eLife, 3, e02403.

Kaya, T. \& Kose, H. (2012) Direct upstream motility in it Eschechia coli.. Biophys. J., 102, 1514-1523.

Kim, S. \& Karrila, S. (2005) Microhydrodynamics. Principles and Selected Applications. Dover Press.

Klein, J., Clapp, A. \& Dickinson, R. B. (2003) Direct measurement of interaction forces between a single bacterium and a flat plate. Journal of Colloid and Interface Science, 261, 379-385.

Leisch, K. A., Pelle, D. W., Dominic, W. \& Lindemann, C. B. (2008) Insights into the mechanism of ADP action on flagellar motility derived from studies on bull sperm. Biophys. J., 95, 472-482.

Lim, S., Ferent, A., Wang, X. S. \& Peskin, C. S. (2008) Dynamics of a closed rod with twist and bend in fluid. SIAM Journal on Scientific Computing, 31, 273-302.

Lindemann, C. B. (1994a) A geometric clutch hypothesis to explain oscillations of the axoneme of cilia and flagella. Journal of Theoretical Biology, 168, 175-189.

Lindemann, C. B. (1994b) A model of flagellar and ciliary functioning which uses the forces transverse to the 
axoneme as the regulator of dynein activation. Cell Motility and Cytoskeleton, 29, 141-154.

Lindemann, C. B. (2002) Geometric Clutch Model Version 3: The Role of The Inner and Outer Arm Dyneins in The Ciliary Beat. Cell Motility and Cytoskeleton, 52, 242-254.

Lindemann, C. B., Orlando, A. \& Kanous, K. S. (1992) The flagellar beat of rat sperm is organized by the interaction of two functionally distinct populations of dynein bridges with a stable central axonemal partition. Journal of Cell Science, 102, 249-260.

Miki, K. \& Clapham, D. E. (2013) Rheotaxis guilds mammalian sperm. Current Biology, 23, 443-452.

Montenegro-Johnson, T. J., Gadêlha, H. \& Smith, D. J. (2015) Spermatozoa scattering by a microchannel feature: an elastohydrodynamic model. Royal Society Open Science, 2, 140475.

Murase, M., Hines, M. \& Blum, J. J. (1989) Properties of an excitable dynein model for bend propagation in cilia and flagella. Journal of Theoretical Biology, 139, 413-430.

Okuno, M. \& Hiramoto, Y. (1979) Direct Measurements Of The Stiffness Of Echinoderm Sperm Flagella. J. Exp. Biol., 79, 235-243.

Olson, S. D., Lim, S. \& Cortez, R. (2013) Modeling the dynamics of an elastic rod with intrinsic curvature and twist using a regularized Stokes formulation. Journal of Computational Physics, 238, 169-187.

Palacci, J., Sacanna, S., A. Abramian, J. B., Hanson, K., Grosberg, A. Y., Pine, D. J. \& Chaikin, P. M. (2015) Artificial rheotaxis. Science Advances, 1, e1400214.

Pozrikidis, C. (1992) Boundary Integral and Singularity Methods for Linearized Viscous Flow. Cambridge University Press.

Psenicka, M., Alavi, S. M. H., Rodina, M., Gela, D., Nebesarova, J. \& Linhart, O. (2007) Morphology and ultrastructure of Siberian sturgeon (Acipenser baerii) spermatozoa using scanning and transmission electron microscopy. Biology of the Cell, 99(2), 103-115.

Riedel-Kruse, I. H., Hilfinger, A., Howard, J. \& Julicher, F. (2007) How molecular motors shape the flagellar beat. HFSP Journal, 1, 192-208.

Rothschild (1963) Non-random distribution of bull spermatozoa in a drop of sperm suspension. Nature, 198, 12211222.

Schmitz-Leisch, K. A. \& Lindemann, C. B. (2004) Direct measurement of the passive stiffness of rat sperm and implications to the mechanism of the calcium response. Cell Motil. Cytoskel., 59, 169-179.

Sharp, J. \& Dickinson, R. B. (2005) Direct evaluation of DLVO theory for predicting long-range forces between a yeast cell and a surface. Langmuir, 21, 8198-8203.

Simons, J., Fauci, L. \& Cortez, R. (2015) A fully three-dimensional model of the interaction of driven elastic filaments in a Stokes flow with applications to sperm motility. Journal of Biomechanics, 48(9, SI), 16391651.

Smith, D. J. (2009) A boundary element regularized Stokeslet method applied to cilia- and flagella-driven flow. Proceedings of the Royal Society A, 465, 3605-3626.

Smith, D. J., Gaffney, E. A., Blake, J. R. \& Kirkman-Brown, J. C. (2009a) Human sperm accumulation near surfaces: a simulation study. Journal of Fluid Mechanics, 621, 289-320.

Smith, D. J., Gaffney, E. A., Gadêlha, H., Kapur, N. \& Kirkman-Brown, J. (2009b) Bend propagation in the flagella of migrating human sperm, and its modulation by viscosity. Cell Motility and the Cytoskeleton, 66, 220-236.

Smith, D. J., Gaffney, E. A., Shum, H., Gadelha, H. \& Kirkman-Brown, J. (2011) Comment on the Article by J. Elgeti, U. B. Kaupp, and G. Gompper: Hydrodynamics of Sperm Cells Near Surfaces. Biophysical Journal, 100(9), 2318-2320.

Spagnolie, S. E. \& Lauga, E. (2010) The optimal elastic flagellum. Physics of Fluids, 22, 031901.

Spagnolie, S. E. \& Lauga, E. (2012) Hydrodynamics of self-propulsion near a boundary: predictions and accuracy of far-field approximations. Journal of Fluid Mechanics, 700, 105-147.

Tornberg, A. K. \& Shelley, M. J. (2004) Simulating the dynamics and interactions of flexible fibers in Stokes flows. Journal of Computational Physics, 196, 8-40.

Tung, C. K., Ardon, F., Roy, A., Koch, D. L., Suarez, S. S. \& Wu, M. (2015) Emergence of upstream swimming 
via a hydrodynamic transition. Physical Review Letters, 114, 108102.

Vernon, G. G. \& Woolley, D. M. (1999) Three-dimensional motion of avian spermatozoa. Cell motility and the cytoskeleton, 42(2), 149-161.

Winet, H., Bernstein, G. S. \& Head, J. (1984) Observations on the response of human spermatozoa to gravity, boundaries and fluid shear. J. Reprod. Fert., 70, 511-523.

Woolley, D. M. (2003) Motility of spermatozoa at surfaces. Reproduction, 126, 259-270.

Woolley, D. M. \& Vernon, G. G. (2001) A Study Of Helical And Planar Waves On Sea Urchin Sperm Flagella, With a Theory Of How They Are Generated. J. Exp. Biol., 204, 1333-1345.

Xu, G., Wilson, K. S., Okamoto, R. J., Shao, J.-Y., Dutcher, S. K. \& Bayly, P. V. (2016) Flexural Rigidity and Shear Stiffness of Flagella Estimated from Induced Bends and Counterbends. Biophyscal Journal, 110(12), 2759-2768. 